\title{
Biologische Beobachtungen bei der Aufzucht von Heringsbrut ${ }^{1}$
}

\author{
Von J. H. S. Blaxter und G. Hempel \\ Marine Laboratory, Aberdeen Biologische Anstalt Helgoland, Helgoland
}

(Mit 6 Abbildungen und 5 Tabellen im Text)

\begin{abstract}
Inhaltsübersicht: I. Einleitung. - II. Befruchtung, Inkubation und Dottersackstadium. 1. Methodik. 2. Ergebnisse. a. Temperaturversuch: Befruchtungs- und Schlüpfrate; Inkubationszeit; Körperlänge; Kiel $\times$ Clyde-Bastarde. b. Salzgehaltsversuch. c. Tageslichtversuch. III. Beobachtungen bei der weiteren Aufzucht der Larven. 1. Allgemeine Aufzuchtbedingungen. 2. Ernährung: Nahrungsangebot; Nahrungsaufnahme und Verdauungsgeschwindigkeit. 3. Wachstum und Entwicklung. 4. Sterblichkeit. - IV. Diskussion. - V. Zusammenfassung. VI. Summary, - VII. Literaturverzeichnis.
\end{abstract}

\section{Einleitung}

Im Frühjahr und Sommer 1960 wurden auf Helgoland die Aufzuchtversuche an Heringsbrut wieder aufgenommen, die durch den Krieg und die Zerstörung der Biologischen Anstalt unterbrochen worden waren (КоттнAus 1939, Sснасн 1939). Die Versuche schließen methodisch unmittelbar an die Arbeiten in Aberdeen an, über die eine zusammenfassende Darstellung von BlaXter (1961) in Vorbereitung ist. Einige biologische und physiologische Beobachtungen, die Blaxter $(1955,1956,1957,1961)$ und Holliday und Blaxter (1960) über die Reaktionen der Brut gegenüber Temperatur, Salzgehalt, Licht und Futter an Heringen des Clyde und - in geringerem Umfang - an Bankheringen der nördlichen Nordsee angestellt hatten, wurden an der normalerweise unter ganz anderen Bedingungen aufwachsenden Brut der Frühjahrsheringe der deutschen Küsten wiederholt. Auch konnten die Untersuchungen über die Modifizierbarkeit der Myomerenzahl, die in Wilhelmshaven (Hempel 1953) und Aberdeen (Blaxter 1957) unabhängig voneinander begonnen worden waren, fortgeführt werden ${ }^{2}$. Dabei bestand die Hoffnung, daß die gute Versorgung mit frischem Seewasser und Wildplankton eine Aufzucht der Heringslarven bis zum Stadium der Wirbelbildung und zur Metamorphose möglich machen würde. Zwar ist die Aufzucht von He-

1) Die Untersuchungen wurden ermöglicht durch Reisestipendien des Department of Agriculture and Fisheries of Scotland und des Bundesministeriums für Ernährung, Landwirtschaft und Forsten und durch Sachbeihilfen der Deutschen Wissenschaftlichen Kommission für Meeresforschung.

2) Über die Ergcbnisse der Myomerenzählungen wird andernorts berichtet werden (Hempel und Blaxter 1961). 
ringsbrut bis zu diesen Stadien schon mehrfach in beschränktem Umfange gelungen (vgl. Zusammenstellung bei BlaXTER 1961), die Versuche fanden aber nie unter kontrollierten Bedingungen statt. Die Helgoländer Versuche haben nun gezeigt, daß auch beim Hering die Aufzucht unter konstanten und reproduzierbaren Bedingungen und in relativ kleinen Zuchtbecken grundsätzlich möglich ist, wenn auch mit hohen Verlusten während der Larvenzeit gerechnet werden muß.

Eine ausführliche Beschreibung der auf Helgoland für die Erbrütung und Aufzucht verwendeten Technik ist nicht erforderlich, da die Methode nur wenig von dem von BLAXTER (1961) beschriebenen Verfahren abweicht.

\section{Befruchtung, Inkubation und Dottersackstadium}

\section{Methodik}

Als Elterntiere dienten Frühjahrslaicher aus der Elbmündung und der Kieler Förde, die dort in stehenden Geräten gefangen worden waren. Ferner wurde das tiefgefrorene Sperma eines am 10. März 1960 im Firth of Clyde, Westschottland, gefangenen Herings entsprechend dem von Blaxter (1955) entwickelten Verfahren verwendet. Auf Grund der an schottischen Heringen gesammelten Erfahrungen wurden die Gonaden aus noch lebenden

Tabelle 1

Länge und meristische Merkmale der für die Erbrütungsversuche verwendeten Elterntiere

\begin{tabular}{|c|c|c|c|c|c|c|c|c|}
\hline 碞 & 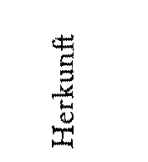 & 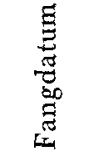 & 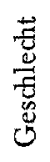 & 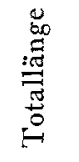 & $\begin{array}{r}\dot{n} \\
\dot{w} \\
\dot{w}\end{array}$ & $\mathrm{D}$ & A & Versuch \\
\hline 1 & Clyde & 10.3 & 8 & 27,3 & 57 & & & Temperatur \\
\hline 2 & Cuxhaven & 26.4 & 웅 & 25,0 & 56 & 18 & 15 & Temperatur + Tageslicht \\
\hline$\overline{3}$ & Cuxhaven & 26.4 & 8 & 27,4 & 56 & 19 & 17 & Temperatur + Tageslicht \\
\hline 4 & Cuxhaven & 26,4 & q & 23,5 & 56 & 18 & 15 & Salzgehalt \\
\hline 5 & Cuxhaven & 26.4 & $\hat{\delta}$ & 25,0 & 57 & 19 & 17 & Salzgehalt \\
\hline 6 & Kiel & 29.4 . & $q$ & 25,3 & 56 & 19 & 17 & Temperatur \\
\hline 7 & Kiel & 29.4 & $\hat{\delta}$ & 24,0 & 57 & 18 & 19 & Temperatur \\
\hline 8 & Kiel & 29.4. & $q$ & 24,3 & 55 & 19 & 15 & Salzgehalt \\
\hline 9 & Kiel & 29.4 & 8 & 21,8 & 56 & 18 & 18 & Salzgehalt \\
\hline 10 & Cuxhaven & 29.4 & $\hat{0}$ & 25,5 & 56 & 18 & 17 & Tageslicht \\
\hline 11 & Cuxhaven & 29.4 & $q$ & 26,0 & 54 & 18 & 18 & Tageslicht \\
\hline
\end{tabular}

Heringen herauspräpariert, trocken in kleinen Gläsern auf Eis aufbewahrt und nach Helgoland gebracht. Dort erfolgte die Befruchtung 8 bis 20 Stunden nach der Präparation. Dies Verfahren hat sich ebenso gut bewährt wie der Transport der Gonaden in situ, in dem der ganze Hering auf Eis gelagert wurde. Jede Versuchsserie wurde mit den Nachkommen eines einzigen Elternpaares durchgeführt. Eine Zusammenstellung der Elterntiere findet sich in Tabelle 1 .

In den Temperaturversuchen erfolgte die Befruchtung in verdünntem Seewasser $(15 \%$ ) in flachen Schalen. Die Eier wurden möglichst schütter (ca. 2-4 Eier $/ \mathrm{cm}^{2}$ ) auf Milchglasscheiben verteilt und anschließend Sperma in das 
Wasser gegeben. Die Temperatur während der Befruchtung war wenig höher als auf dem Laichplatz, d. h. sie lag bei $10-12^{0} \mathrm{C}$. In diesem Wasser verblieben die Glasplatten mit den Eiern etwa 5 Stunden, dann wurden sie in die endgültigen Becken und in langsam fließendes Seewasser von ca. 33\% Salzgehalt überführt. Der erste Versuch, bei dem die Befruchtung auf FK „Uthörn" während der Überfahrt von Cuxhaven nach Helgoland erfolgte, war ein vollständiger Fehlschlag. Die große Mehrzahl der Eier blieb unbefruchtet, oder die Frühentwicklung des Keimes verlief abnorm ${ }^{3}$ ). Nur 1 bis $2 \%$ der Eier erbrachte lebensfähige Larven. Es wäre interessant festzustellen, ob die Vibrationen des Schiffes einen ungünstigen Einfluß auf die Befruchtung oder auf die frühe Embryonalentwicklung haben.

Die Erbrütung wurde in mehreren Parallelversuchen durchgeführt, in denen die Eier jeweils verschiedenen Bedingungen ausgesetzt waren. In der ersten Versuchsserie erfolgte die Erbrütung bei verschiedenen Temperaturen (ca. $5^{0}, 8^{0}, 11^{0}, 14^{\circ} \mathrm{C}$ ). Sie wurde mehrmals an Eiern verschiedener Herkunft wiederholt, und in ihr wurden auch die Kiel $\times$ Clyde-Bastarde mit der Brut von Kiel $\times$ Kiel verglichen. Die auf Milchglasscheiben festgeklebten Eier hingen zur Erbrütung in 8 schwarzgestrichenen Steinzeugbedken mit 1201 Fassungsvermögen. Die Becken standen in einem temperaturkonstanten Raum, dessen Lufttemperatur auf $3-4^{0} \mathrm{C}$ eingestellt war. Jedes der Aufzuchtbecken wurde aus einem kleineren Glasgefäß (101) mit vortemperiertem, unfiltriertem Seewasser gespeist. Diese Zulaufbecken waren an die Hauptseewasserleitung des Instituts angeschlossen. Aquarienheizer erwärmten in ihnen das zulaufende Wasser nach Bedarf, bevor es auf den Boden des Zuchtbeckens geleitet wurde. Die Heizung wurde durch Kontaktthermometer, die in den Zuchtbecken hingen, gesteuert. Der Wasserzulauf betrug 0,2-0,8 1/Min. Der Ablauf aus den Becken lag in der Mitte der Wasseroberfläche. Er war durch Nylongaze gesichert, so daß keine Larven oder Nährtiere verlorengehen konnten. Die Nylongaze verhinderte aber auch, daß sich die Oberfläche des Beckens selbsttätig reinigte. So bildete sich schnell eine Kahmhaut, auch sammelte sich Staub auf der Wasseroberfläche. Jeden zweiten Tag mußte die Oberfläche gereinigt werden, indem man kurzfristig die Nylongaze vom Abfluß entfernte. Währenddessen wurden Larven, die sich dem Abfluß näherten, mit einer Pipette in entferntere Teile des Beckens zurückgesetzt. Durch die bei der Erwärmung auftretenden Luftblasen im Wasser war es schwierig, einen gleichmäßigen Wasserfluß durch das Hebersystem zu erzielen. Hierdurch traten in den auf $14^{\circ}$ und $8^{\circ} \mathrm{C}$ eingestellten Becken anfangs Temperaturschwankungen auf. Schließlich stellten wir die Heizgefäße jeweils in die Erbrütungsbecken und ließen das erwärmte Wasser aus ihnen durch einen Heber auf den Grund des Beckens laufen. Obwohl der Zulauf am Becken-Boden, der Ablauf an der Oberfläche lag, traten in den am stärksten erwärmten Becken vertikale Temperaturunterschiede von $1^{\circ} \mathrm{C}$ auf. Stets wurde die Temperatur in derjenigen Wasserschicht registriert, in der die Glasplatten mit den Eiern hingen. Die Eier wurden im Dunklen erbrütet. Nach dem Schlüpfen waren die Larven der diffusen Beleuchtung (Glühfaden-Röhrenlampen) des Raumes 16 Stunden

3) Spätere Kontrollversuche im Labor zeigten, daf3 Eier, denen man eine Unterlage zum Festheften bietet, mehrere Furchungen durchmachen können, ohne daß sie befruchtet werden. Die Zellen dieser unbefruchteten Eier sind oft blasig aufgetrieben und bilden einen unregelmäßigen Zellhaufen. Zahlreiche Eier des ersten Befruchtungsversuches ähnelten solchen abnormen Entwicklungen. VolODIN (1956) hat die parthenogenetische Entwicklung von Heringseiern eingehender beschrieben. 
täglich ausgesetzt. An der Wasseroberfläche betrug die Lichtintensität etwa 100 Lux. - Die zweite Versuchsserie war dem Einfluß des Salz gehalts ( 5 bis $50 \%$ ) auf die Heringsbrut gewidmet. Die Eier klebten in diesem Fall auf dem Boden von 3-1-Gläsern, die mit Wasser des jeweils gewünschten Salzgehalts in einem auf $10^{\circ} \mathrm{C}$ eingestellten temperaturkonstanten Raum standen. Die Befruchtung wurde entweder in 15\% Salzgehalt durchgeführt, oder es wurde für sie Wasser der gleichen Salinität wie bei der Aufzucht verwendet. Jeden zweiten oder dritten Tag wurden die Gläser mit frischem, vortemperiertem Seewasser neu gefüllt. Die verschiedenen Salzgehalte wurden durch Verdünnung von natürlichem Seewasser mit Süßwasser (Ionen-Austauscher) oder durch Anreicherung des natürlichen Seewassers mit den wichtigsten Salzen erzeugt und mit dem Aräometer unter Verwendung von Gillbrichrs (1959) Fluchtentafel kontrolliert. - Die Eier der dritten Versuchsserie waren dem Tageslicht ausgesetzt, sie sollten das Ausgangsmaterial für Versuche über die Bedeutung unterschiedlicher Nahrung auf die Überlebensrate liefern. Die Eier wurden - ebenfalls auf Milchglasscheiben - in 3 100-1-Eternitbecken in einem Kellerraum („Kulturraum“) gehalten, dessen hochgelegene Fenster nur am frühen Morgen Sonne in die Becken fallen ließen. Hier wurde die Wassertemperatur nicht konstant gehalten, sie schwankte mit der Raumtemperatur des zufließenden Wassers.

$\mathrm{Zu}$ dem Zeitpunkt, in dem etwa $50 \%$ der Larven geschlüpft waren („Ende der Inkubationszeit"), wurden den Becken und Gläsern jeweils etwa 50 Larven entnommen. Nach Betäubung mit 2\% Urethan konnten die Larven in gut gestrecktem Zustand konserviert werden ( $2 \%$ Formol-Seewasser $\left.)^{4}\right)$. War das Schlüpfen beendet, wurden die Platten aus den Becken entfernt. Zur Bestimmung der Befruchtungs- und Schlüpfraten wurden anschließend die verlassenen Eihäute, die abgestorbenen und die nicht befruchteten Eier auf den Platten und auf dem Boden der Gläser (Salzgehaltversuche) ausgezählt.

Der Salzgehalt im Temperatur- und Tageslichtversuch änderte sich nur in engen Grenzen, etwa entsprechend den Salinitätsschwankungen auf Helgoland Reede. Auf eine genaue Ermittlung der Dauer des Dottersackstadiums in Abhängigkeit von Temperatur und Salzgehalt wurde bei diesen Versuchen verzichtet. Etwa 60 Tagesgrade nach dem Schlüpfen war allgemein der Dotter resorbiert. Um diese Zeit wurde bei einigen Versuchen jeweils eine zweite Probe von 50 Larven konserviert.

Die Larven wurden später mit dem Meßokular eines Stereomikroskops ausgemessen. Bestimmt wurde die Totallänge von der Schnauzenspitze bis zum Hinterrand der Primordialflosse, die Körperhöhe im Bereich des Dottersacks und die Dicke des Dottersacks (gemessen senkrecht zur Körperachse der auf der Seite liegenden Larve).

Alle Längenangaben in dieser Arbeit beziehen sich auf Proben, die etwa einen Monat nach der Fixierung gemessen wurden. Unfixierte, mit Urethan betäubte Dottersacklarven waren vor der Fixierung um etwa $10 \%$ länger als nach einmonatigem Aufenthalt in Formalin. Larven, die vier. Monate später nochmals gemessen wurden, waren um weitere $4 \%$ geschrumpft. Für alle Vergleiche zwischen Larvenproben verschiedener Herkunft ist es daher

4) Die von uns angewandte Methode der Probennahme ist nicht voll befriedigend. Die eutnommenen Larven sind - bezogen auf den Schlüpftermin - nicht völlig gleichaltrig, auch kann in beschränktem Maße beim Fangen der Larven mit der Pipette eine Selektion stattfinden. Demnächst soll geprüft werden, ob bezüglich der Körpermaße Unterschiede zwischen den zuerst, später und zuletzt geschlüpften Larven einer Eierprobe bestehen. 


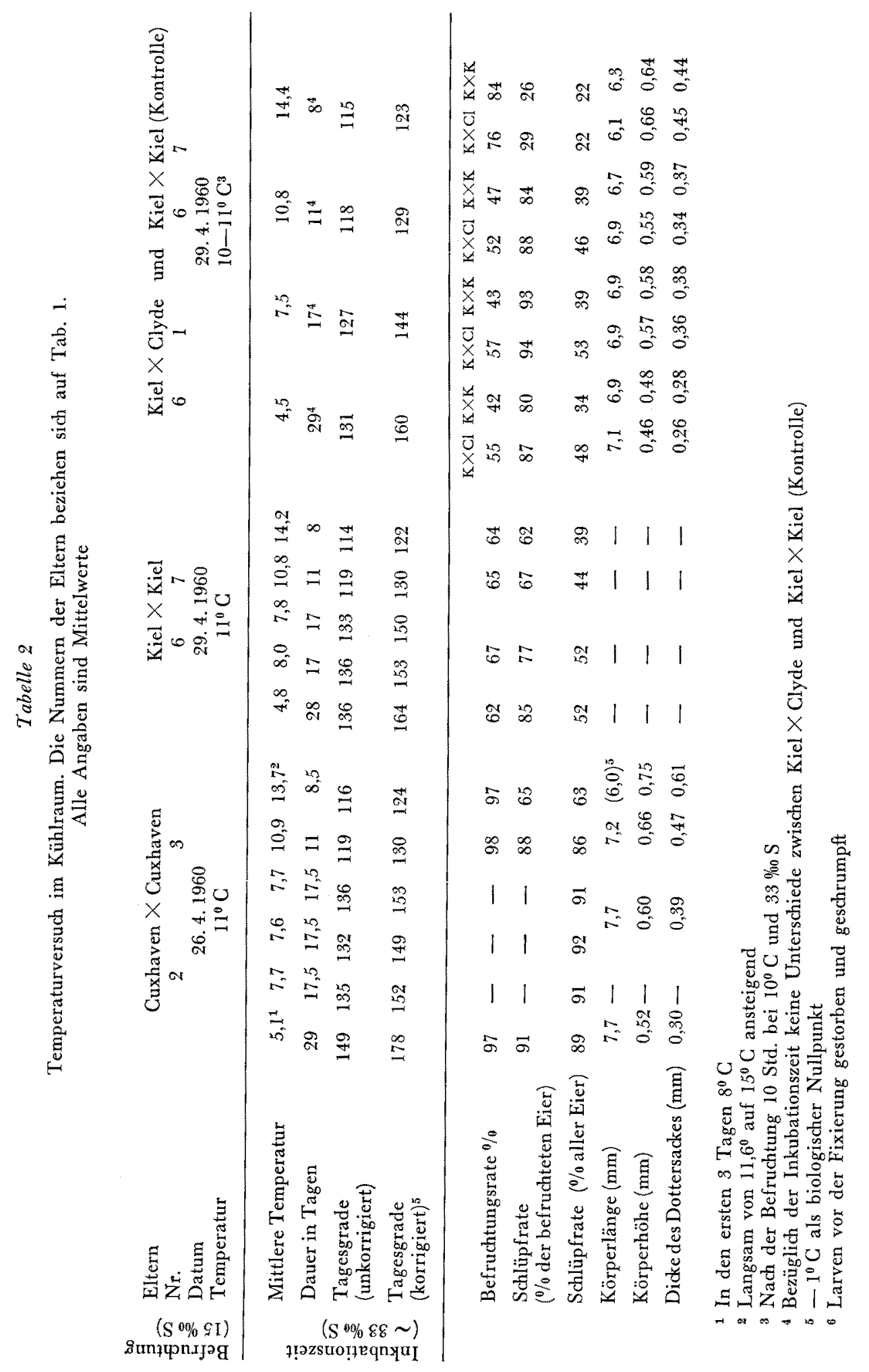


wichtig, daß sie bezüglich der Stärke des Fixierungsmittels und der Dauer der Fixierung recht genau übereinstimmen.

Herrn Dr. KrefFr, Institut für Seefischerei, Hamburg, und Herrn Dr. Thurow, Institut für Meereskunde, Kiel, danken wir herzlich für ihre freundliche Unterstützung bei der Beschaffung der Laichheringe. Die Larvenmessungen verdanken wir großenteils Frau Dr. HemPEI. Zu großem Dank sind wir mehreren Mitarbeitern der Biologischen Anstalt Helgoland verpflichtet, vor allem Herrn J. Herzog und Herrn W. Krüss, die uns auch unter ungünstigen Bedingungen mit Plankton versorgten, und Herm H. Trekel für seine Hilfe beim Aufbau der Erbrütungscinrichtungen. Das Aquarium San Franzisko überlieB uns freundlicherweise eine größere Menge Artemia-Eier.

\section{Ergebnisse}

\section{a. Temperaturversuch}

Die Tabelle 2 faßt die Ergebnisse der Temperaturversuche zusammen, die sich an die Befruchtungen Cuxhaven $\times$ Cuxhaven 26.4.60, Kiel $\times$ Kiel und Kiel $\times$ Clyde 29. 4. 60 anschlossen. Die Kiel $\times$ Kiel-Brut wurde in zwei Parallelreihen gehalten, von denen die eine als Kontrolle genau den gleichen Bedingungen wie die Kiel x Clyde-Brut ausgesetzt wurde.

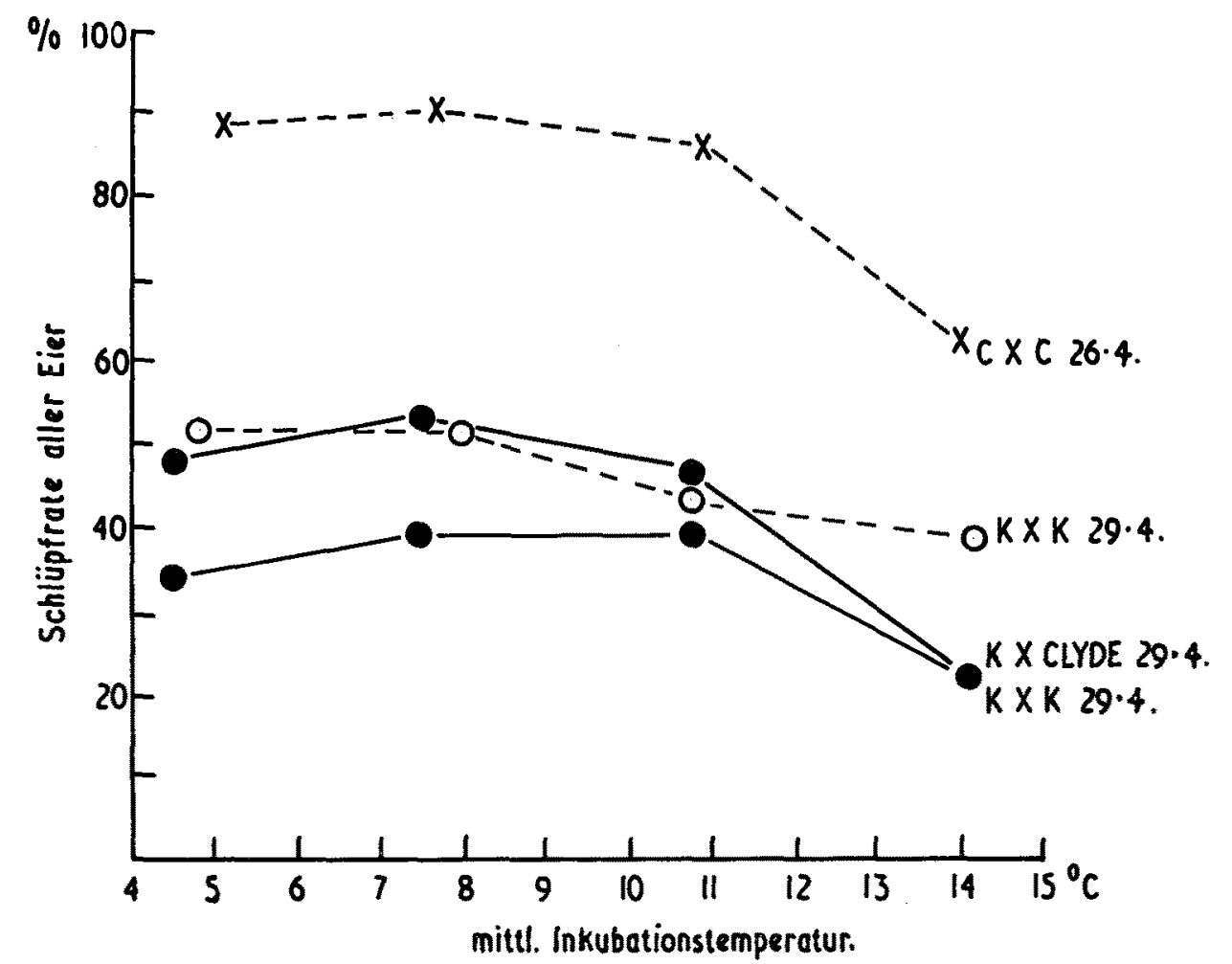

Abb. 1. Temperaturabhängigkeit der Schlüpfrate bei verschiedenen Erbrütungsversuchen. Eier von Cuxhavener $(\mathrm{C})$ und Kieler (K) Heringen

Befruchtungs- und Schlüpfrate: Im Gegensatz zu dem Vorversuch vom 20. April lag die Befruchtungsrate bei den Elbheringseiern vom 26. April sehr hoch; bei den Kieler Heringen war der Befruchtungserfolg 
geringer. Während von den Guxhavener Eiern $97-98 \%$ befruchtet wurden, waren es bei den Kieler Eiern nur etwa $40-60 \%$. Die Ursache für diesen Unterschied mag in dem weiteren Transport der Kieler Gameten zu suchen sein. Bei der Betrachtung der Befruchtungsraten in Tabelle 2 ist zu beachten, daß die Temperatur während der Befruchtung innerhalb der einzelnen Versuche gleich war und die Eier erst später unterschiedlichen Temperaturen ausgesetzt wurden.

Die relativ hohen Befruchtungsraten der später bei $14^{\circ} \mathrm{C}$ erbrüteten Kiel $\times$ Kiel- und Kiel $\times$ Clyde-Eier sind möglicherweise durch eine falsche Beurteilung der abgestorbenen Eier zu erklären, indem manche tote Eier fälschlich für befruchtet gehalten wurden (s. o. Fußnote zu S. 262).

Die Schlüpfrate in Prozent aller Eier ist für den Temperaturbereich von $5^{\circ}$ bis $11^{\circ} \mathrm{C}$ innerhalb der einzelnen Versuche etwa gleich. In den meisten Fällen zeichnet sich ein schwaches Maximum bei $8^{\circ} \mathrm{C}$ ab, d. h., bei derjenigen Versuchstemperatur, die den Verhältnissen auf dem Laichplatz am nächsten kommt. Bei den in $14^{\circ} \mathrm{C}$ warmem Wasser enbrüteten Eiern war dagegen die Schlüpfrate deutlich geringer (Abb. 1). $\mathrm{O}_{2}$-Mangel, $\mathrm{CO}_{2}$-Vergiftung oder Schädigung durch bei hoher Temperatur gehäuft auftretende Abbauprodukte wurden durch den ständigen Wasserzufluß zumindest stark eingeschränkt; vermutlich kommt die Erbrütungstemperatur von $14-15^{\circ} \mathrm{C}$ der oberen Grenze für die normale Entwicklung der Embryonen des Frühjahrsherings nahe. Allerdings hat KupfFer (1878) noch bei $14-19^{\circ} \mathrm{C}$ Eier des Schleiherings erfolgreich erbrütet. In der westlichen Ostsee (Mryer 1878a) und in der Elbmündung erfolgt das Laichen aber normalerweise bei Temperaturen unter oder um $10^{\circ} \mathrm{C}$. Beim Clyde-Hering beschreibt Blaxter $(1957,1961)$ eine ähnliche Empfindlichkeit der Eier gegen höhere Temperaturen, während Herbstheringe der nördlichen Nordsee, die in wärmerem Wasser laichen, nicht derartig von einer hohen Erbrütungstemperatur beeinträchtigt werden.

Inkubationszeit: In Abb. 2 ist die Abhängigkeit der Inkubationszeit von der Temperatur dargestellt. Zum Vergleich dienen einige Werte aus Blaxters Erbrütungsversuchen an Clyde- und Bank-Heringen. Bei niedriger Temperatur war die Zeit zwischen Befruchtung und Schlüpfen bei den schottischen Heringen etwas kürzer. In unseren Versuchen stimmten, gleiche Erbrütungstemperatur vorausgesetzt, die Inkubationszeiten für die Elbheringe mit denjenigen der Kieler Heringe recht gut überein. Bei der Berechnung der Tagesgrade (mittlere Erbrütungstemperatur $X$ Inkubationstage) (Abb. 3) wurde willkürlich $0^{\circ} \mathrm{C}$ als der biologische Nullpunkt angesetzt. Nach BLAXTER (1957) beträgt diejenige Temperatur, bei der die Entwicklung des Embryos unendlich langsam wäre, für den Clyde-Hering etwa $-1,3^{0} \mathrm{C}$. Für den norwegischen Frühjahrshering berechnete Soleım (1942) - 1,20 C. Der exakten Bestimmung der Inkubationszeit steht entgegen, daß der Zeitpunkt, zu dem $50 \%$ der Larven geschlüpft sind, nur schwer genau zu bestimmen ist. So haftet auch der Berechnung der Tagesgrade eine gewisse Unsicherheit an.

Körperläng e: MeYer (1878b) hatte beobachtet, daß Heringslarven, die in kaltem Wasser erbrütet worden waren, beim Schlüpfen im Durchschnitt länger und dotterärmer waren als warm erbrütete. BLAXTER (1957) fand dagegen nur einen geringen Temperatureinfluß auf die Körperlänge frischgeschlüpfter Larven. In Tabelle 2 und Abb. 4 sind die mittleren Körperlängen der Larven und die Durchmesser der Dottersäcke für die einzelnen bei verschiedener Temperatur erbrüteten Proben dargestellt. Unsere Versuche er- 
gaben ein starkes Absinken der Körperlänge mit steigender Temperatur. Die Größe des Dottersackes ist dagegen positiv mit der Erbrütungstemperatur korreliert.

Die Larven waren im Durchschnitt größer als die von BtAXTer (1957) erbrüteten Larven des Nordseebankherings, während sich klare Unterschiede zu den Clyde-Larven nicht ergaben. Die geringere Körpergröße der Bankherings-Larven dürfte auf die weitaus höhere Fruchtbarkeit des Bankherings zurückzuführen sein, die eine geringere Größe der einzelnen Eier bedingt. Dem scheint $\mathrm{zu}$ widersprechen, daß Meyer (1878b) größere Larven von Herbstlaichern der Ostsee gewann als von Frühjahrslaichern, obwohl nach KäNDLER und DutT (1958) der Herbsthering der Ostsee fruchtbarer als der Frühjahrshering ist. Schwer zu verstehen ist, warum die Larven aus der

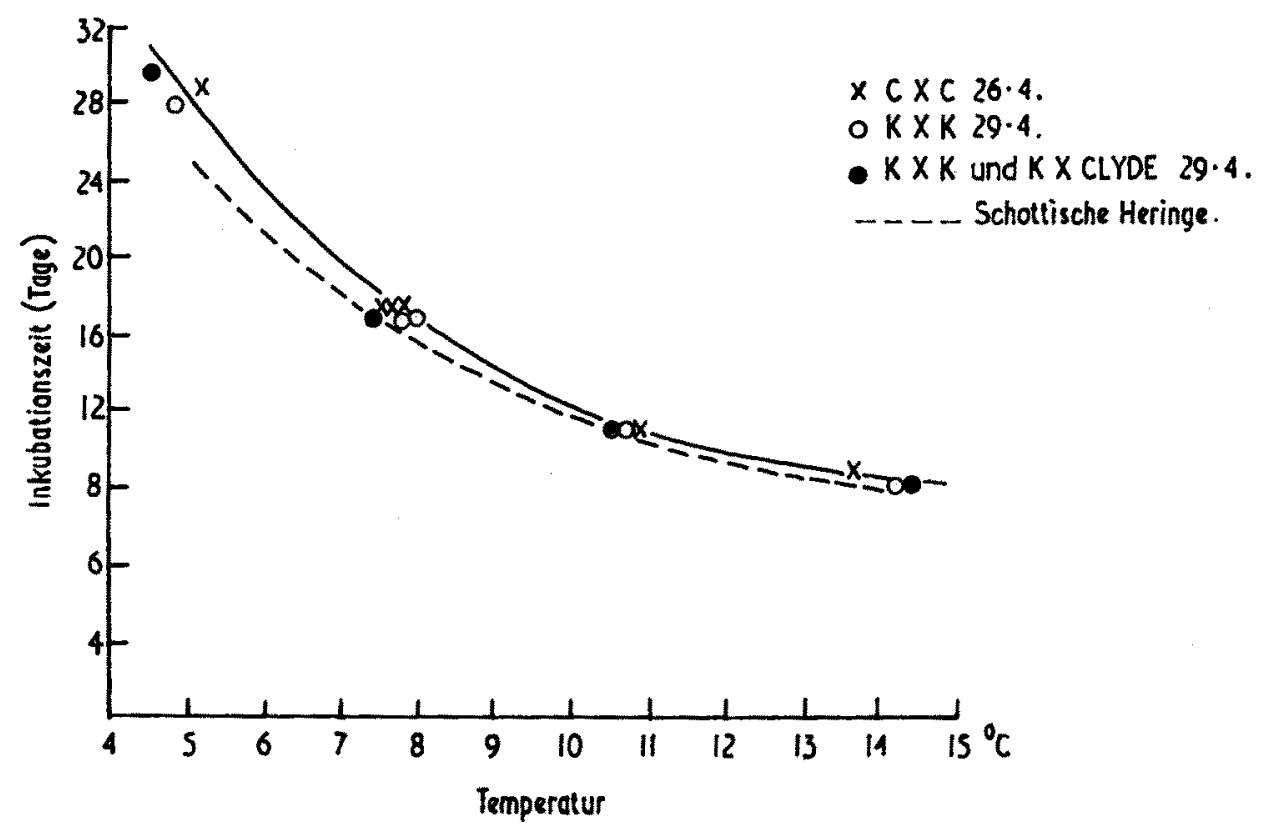

Abb. 2. Temperaturabhängigkeit der Inkubationszeit bei Heringseiern verschiedener Herkunft. Schottische Heringe nach BLAXTeR (1956)

Cuxhaven $\times$ Cuxhaven-Befruchtung vom 26.4. größer waren als die Kieler Larven. Bedeutende Unterschiede in der Körpergröße der Eltern lagen nicht vor.

Von besonderem biologischen Interesse ist die Körpergröße der Larven am Ende des Dottersackstadiums. Sie liefert uns ein Maß für die Ausnutzung des Dotters zum Aufbau körpereigener Substanz unter den jeweils herrschenden Aufzuchtbedingungen. Die am Ende des Dottersackstadiums fixierten Larven der Cuxhaven $\times$ Cuxhaven-Befruchtung vom 26. 4.60 zeigten nicht die gleiche Temperaturabhängigkeit wie die unmittelbar nach dem Schlüpfen entnommenen Proben. Während des Dottersackstadiums wuchsen die bei $8^{0} \mathrm{C}$ lebenden Larven am schnellsten (ca. $1 \mathrm{~mm}$ in einer Woche) und erreichten die größte mittlere Körperlänge. Dabei wurde gegen Ende des Dottersackstadiums die Temperatur in den wärmeren Becken gesenkt, in kälteren erhöht, so daß die Temperaturmittel während des Dottersackstadiums nicht ganz so stark differierten wie während der Inkubationszeit. 
Im Rahmen des internationalen Heringslarven-Programms war die Frage aufgetaucht, ob junge Heringslarven durch die Maschen des Gulf III-Sampler entkommen können. Die lichte Weite der Maschen des meistgebrauchten Metallnetzes (40 Maschen/inch) beträgt $0,3-$ $0,4 \mathrm{~mm}$, sie ist weit kleiner als der größte Körperquerschnitt der Heringslarven. Die Ausmessung der Körperhöhe im Bereich des Dottersacks hatte bei frischgeschlüpften Larven Werte von $0,5-0,7 \mathrm{~mm}$ ergeben; der abgeplattete Kopf von Larven, die den Dotter gerade resorbiert haben, ist etwa $0,7 \mathrm{~mm}$ breit.

$\mathrm{Kiel} \times \mathrm{Clyde}-\mathrm{B}$ astarde: Die Erbrütungsversuche ergaben keine auffallenden Unterschiede zwischen den Kiel $\times$ Kiel- und Kiel $\times$ Glyde-Larven. Der Befruchtungserfolg des Clyde-Heringsperma war dem des Kieler Sperma

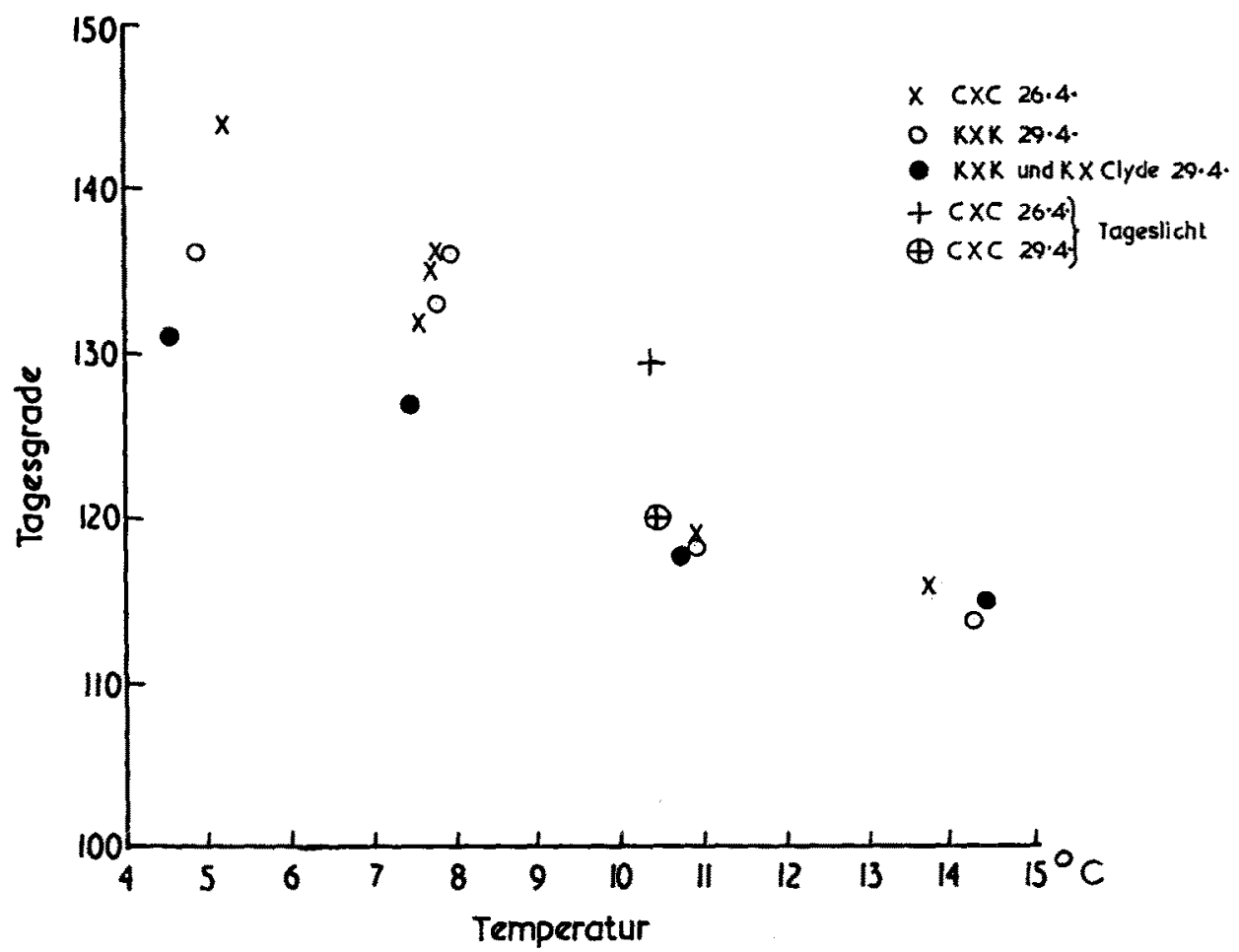

Abb. 3. Anzahl der Tagesgrade (mittlere Inkubationszeif $X$ mittlere Erbrütungstemperatur in ${ }^{\circ} \mathrm{C}$ ) in Abhängigkeit von der Erbrütungstemperatur.

gleich oder sogar etwas besser, was auf die Brauchbarkeit des Tiefgefrierverfahrens hinweist. Auch die Embryonal-Entwicklung verlief bei den $\mathrm{Ba}$ starden normal. Inkubationszeit, Schlüpfrate und Larvengröße waren die gleiche wie bei den Kontrollen.

\section{b. Salzgehaltsversuch}

Die bisherigen experimentellen Arbeiten über den Einfluß des Salzgehalts auf die Heringsbrut bezogen sich fast ausschließlich auf die Bedeutung der Salinität für die Ubberlebensrate der Eier und jungen Larven (vgl. BLAXTER und Holliday 1960). Abb. $4^{5}$ ) zeigt nun, daß eine deutliche Abhängigkeit der

5) Die beiden einzelnen Kreuze im Salzgehaltsversuch gehören Proben an, die erst vier Monate nach der Fixicrung gemessen wurden. Es ist anzunehmen, daß die niedrigen Mittelwerte dieser Proben durch die Schrumpfung der Larven bei langem Aufenthalt in Formalin bedingt sind (s. S. 263). 

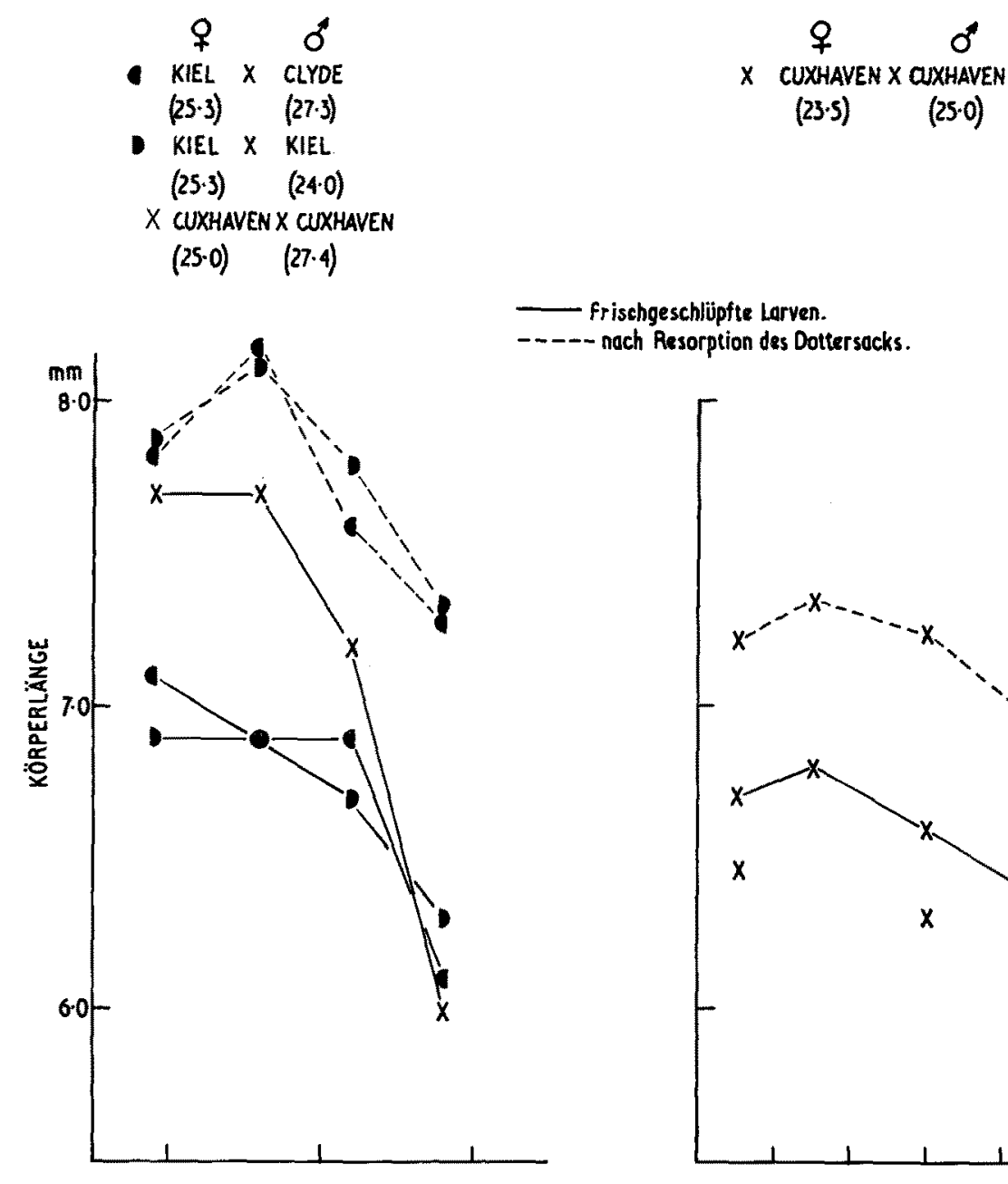

KIEL $X$ KIEL

(25.3) (24.0)

$(25.0) \quad(27.4)$

Frischgeschlüpfte Larven.

nach Resorption des Doltersocks.
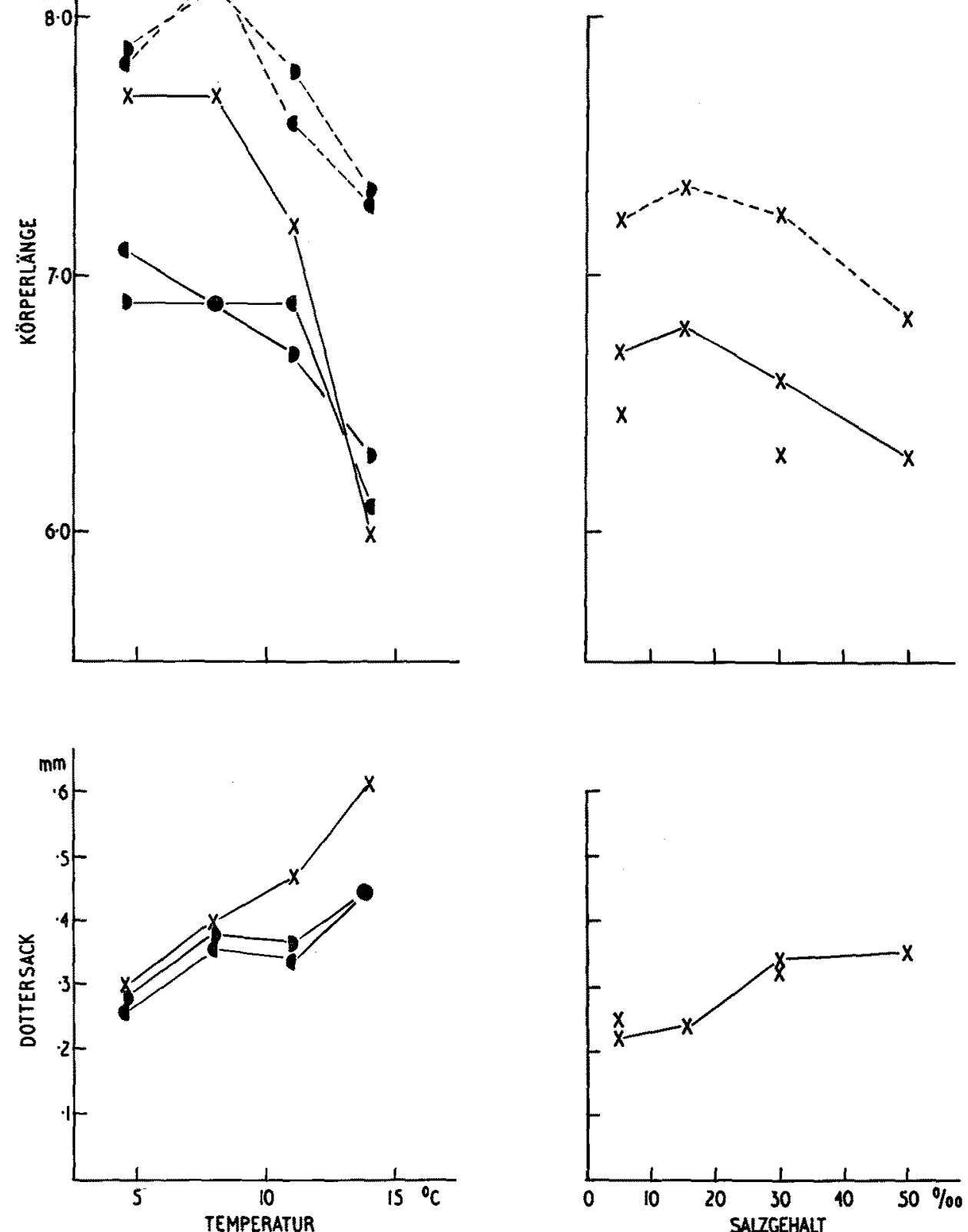

Abb. 4. Körperlänge und Dottersackdurchmesser frischgeschlüpfter Larven in Abhängigkeit von Temperatur und Salzgehalt während der Erbrütung 
Kondition der jungen Larven von dem Salzgehalt, in dem sie erbrütet und während der Inkubationszeit gehalten wurden, besteht. Diejenigen Larven, die bei $15 \%$ erbrütet wurden, waren am größten und dotterärmsten. Bei niedrigerem wie auch bei sehr hohem Salzgehalt schlüpften dagegen kleinere Larven. Ihr Dottersack war zwar größer als bei den 15\%0-Larven, er lieferte aber unter den ungünstigen Bedingungen hohen Salzgehalts nur einen relativ geringen Zuwachs der Körperlänge während des Dottersackstadiums. So kam es, daß auch nach Resorption des Dotters die bei $15 \%$ erbrüteten Larven am längsten waren. Es wird zu prüfen sein, ob es sich hierbei um eine spezielle Anpassung der Küstenheringe an die Salinitätsverhältnisse ihrer Laichplätze handelt, oder ob eine allgemeine Beziehung vorliegt, die durch die Isotonie des Herings bei etwa $15 \%$ bedingt sein könnte.

$$
\text { c. Tageslicht-Versuch }
$$

Auch in diesem Versuch lagen bei der Befruchtung die gleichen Bedingungen vor wie beim Temperaturversuch. Das erste Bedken wurde mit Eiern der Cuxhavener Heringe vom 26. 4., das andere mit solchen vom 29.4. beschickt. Die Anzahl der Tagesgrade war in den zeitweilig dem Sonnenlicht ausgesetzten Becken nicht deutlich von den im Hauptversuch gewonnenen Werten verschieden. Die Befruchtungs- und Schlüpfraten lagen hoch, sie unterschieden sich damit aber nicht von den Ergebnissen, die im Hauptversuch bei ähnlicher Temperatur erzielt wurden.

\section{Beobachtungen bei der weiteren Aufzucht der Larven}

Mit dem Ende des Dottersackstadiums waren die Versuche über die Temperatur- und Salinitätsabhängigkeit der Myomerenzahl und der Körpergröße abgeschlossen. In den folgenden Monaten bemühten wir uns, eine größere Anzahl von Heringslarven bis zur Ausbildung der Wirbelsäule und der Metamorphose am Leben zu erhalten. In einer Reihe von Parallelversuchen wollten wir dabei verschiedene Ernährungs- und Belichtungsverhältnisse in ihrem Einfluß auf die Überlebensrate vergleichen.

\section{Allgemeine Aufzuchtbedingungen}

Die meisten Larven verblieben in dem Becken, in dem sie aus dem Ei geschlüpft waren, d. h. in den 8 künstlich beleuchteten Becken des temperaturkonstanten Raumes (Kühlraum) und den 3 natürlich beleuchteten Becken des Kulturraumes. Im Kühlraum wurde die Temperatur schrittweise erhöht, im Kulturraum stieg sie stärker an. Abb. 5 gibt die Wochenmittel für die Temperaturen der wichtigsten Becken im Kühlraum wieder. In den KühlraumBecken herrschte ein ständiger Wasserdurchfluß. Gelegentliche Kontrollen zeigten, daß sie mit Sauerstoff gesättigt waren. In den Kulturraum-Becken wurde zeitweilig nur jeden 2. Tag etwa $1 / 4$ des Wassers erneuert. Zweifellos trug der mit der Zeit sich entwickelnde Algenbewuchs der Becken zu ihrer Belüftung bei.

Um zu vermeiden, daß sich die Larven in einer Zone optimaler Helligkeit konzentrieren könnten, waren die Röhrenlampen im Kühlraum so angebracht, daß sie die Beckenoberfläche möglichst gleichmäßig und diffus beleuchteten. In 


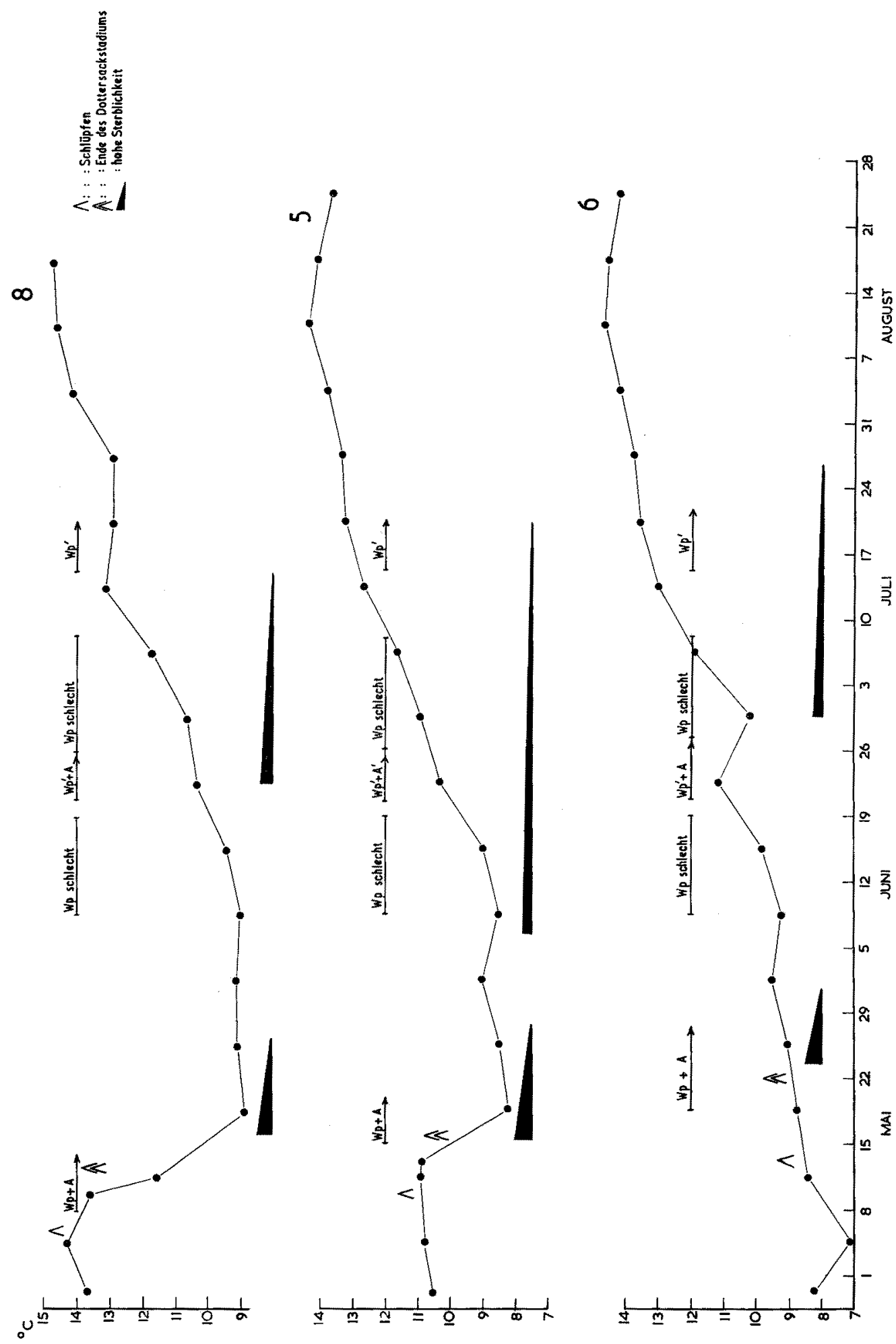

Abb. 5. Die Anderungen der Temperatur (Wochenmittel) während der Aufzuchtversuche in den wichtigsten Kühlraum-Becken. Das Nahrungsangebot und die Zeiten hoher Sterblichkeit in den einzelnen Becken sind eingetragen. $W_{p}=$ Wildplankton, $W_{p}^{\prime}=$ Wildplankton (ungesiebt), $\mathrm{A}=$ frischgeschlüpfte Artemia-Nauplien, $\mathrm{A}^{\prime}=$ ältere Artemia-Nauplien 
den Kulturraum-Becken erwies sich direkter Sonnenschein als ungünstig: Während der Stunden, in denen die Sonne schräg auf die Beckenwand und den Beckenboden fiel, standen die Larven mit dem Kopf zur beleuchteten Wand oder zum Boden. Das gleiche Verhalten ließ sich im Kühlraum mit einem scharfen Lichtstrahl auslösen.

\title{
2. Ernährung
}

a. Nahrungsangebot. Blaxter (1961) hat eine Zusammenstellung der Nahrungsorganismen gegeben, die bei früheren Aufzuchtversuchen verwendet worden sind. Er selbst hatte die besten Erfahrungen mit Balanus-Larven gemacht, während Artemia-Nauplien für die frühen Larvenstadien des Herings ungeeignet zu sein schienen. Wildplankton stand in Aberdeen nicht in ausreichender Menge zur Verfügung. Zur Zeit der Helgoländer Versuche hatte Balanus bereits abgelaicht, statt dessen wurden in beschränktem Umfang Mytilus-Larven gezüchtet, nachdem die Elterntiere durch KCl-Injektion zum Ablaichen gebracht worden waren (VLASBLOM 1959). Die wichtigste Nahrung bildeten aber Artemia-Nauplien und Wildplankton, das ein- bis zweimal

Tabelle 3

Einige Analysen des verfütterten Wildplanktons

Die innerhalb der Proben häufigen Formen sind kursiv gesetzt

\author{
3.6. 60 Copepoden-Nauplien, Chaetoceras \\ 7. 6.60 Polychaeten-Larven, Acartia, Copepoden-Nauplien, Gastropoden-Larven, T emora \\ Rhizosolenia \\ 25. 6. 60 Acartia, Podon, Evadne, Noctiluca, Polychaeten-Larven, Biddulphia sinensis, \\ Rhizosolenia \\ 30.6.60 Temora, Acartia, Noctiluca, Evadne, Podon \\ 2.7.60 Temora, Noctiluca, Acartia, Rhizosolenia, Biddulphia sinensis, Eucampia zoodiacus \\ 8.7.60 Acartia, Paracalanus, Pseudocalanus, Phtisica marina, Temora \\ 15.7.60 Acartia, Paracalanus, Pseudocalanus \\ 23.7.60 Temora
}

täglich auf der Reede von Helgoland mit feinen Planktonnetzen gefangen worden war. Während der ersten zwei Monate wurde das Plankton nach dem Fang durchgesiebt, um größere Räuber zu entfernen; später war diese Vorsichtsmaßregel nicht mehr erforderlich. Die Planktondichte schwankte im Verlauf der Versuche erheblich. Zeitweilig traten unerwünschte Formen wie Phaeocystis und Noctiluca in großer Menge auf. Die Planktonfänge wurden dann für einige Stunden in hohen Glasbecken gehalten, so daß sich Phaeocystis absetzen oder sich wie Noctiluca an der Oberfläche sammeln konnte. Das Zooplankton, das mit Licht angelockt wurde, konnte dann aus den mittleren Wasserschichten abgesaugt werden. Leider war der Bestand an brauchbarem Zooplankton in den Zeiten üppiger Phytoplankton-Wucherungen oft so gering, daß es fraglich war, ob die Zuchtbecken stets mit ausreichenden Mengen geeigneten Planktons versorgt wurden. Zu Beginn der Versuche herrschten Polychaeten- (besonders Polydora) und Copepoden-Larven sowie Rotatorien im Plankton vor, später dominierten die älteren Copepoden. Vom Juni an wurde das Plankton gelegentlich genauer untersucht: In Tabelle 3 ist die $\mathrm{Zu}$ sammensetzung einiger Planktonproben wiedergegeben. 
Um festzustellen, welche Nahrung für die Larven am besten geeignet ist, wurden die einzelnen Becken mit unterschiedlichem Futter beschickt:

\section{Wildplankton}

Artemia-Nauplien

Wildplankton + Artemia-Nauplien

Wildplankton + Mytilus-Trochophoren

Die beste Uberlebensrate ergab sich bei Verwendung von Wildplankton + Artemia. Offenbar bildeten die Artemia-Nauplien ein geeignetes Zusatzfutter, wenn das Wildplankton sehr arm war. Die Larven, die später die Metamorphose erreichten, waren anfangs mit feinem Wildplankton + Artemia, später als - sie $>20 \mathrm{~mm}$ lang waren - ausschließlich mit ungesiebtem Wildplankton gefüttert worden.

Meyer (1878b) fütterte seine Larven aussdhließlich mit Wildplankton, er fand in Zeiten geringer Planktondichte hohe Sterblichkeit und geringes Wachstum. Nachdem er auf cin Durchsieben des Planktons verzichtet und damit das Mengenangebot verbessert hatte, wuchsen die Larven sehr schnell und holten die gleichzeitig geschlüpften Larven des freien Wassers im Wachstum wieder ein.

In einem der Becken wurden große Mengen Wildplankton geboten, als die Heringslarven gerade das Ende des Dottersackstadiums erreicht hatten. Hier war die Sterblichkeit anfangs erheblich geringer als in den anderen Bedken. Aber dieser Erfolg blieb nicht bestehen, als das Plankton infolge der Phaeocystis-Wucherung schlechter wurde.

Im Gegensatz zu den Befunden von Dannevig und Hansen (1952), McMynn und Hoar (1953) und Kurata (1959) gelang es uns, Larven vom Dottersackstadium bis zu einer Länge von $20 \mathrm{~mm}$ mit Artemia aufzuziehen. Wenn es auch nicht ausgeschlossen ist, daß die Larven sehr geringe Mengen anderer Nahrung in dem zufließenden, unfiltrierten Seewasser fanden, so bildeten doch Artemia-Nauplien hier die einzig wichtige Nahrung. In den meisten Fällen wurden die Artemia-Nauplien am ersten Tage nach Verlassen ihres Eies verfüttert. Nur Becken 6 erhielt Artemia-Larven, die 3 Tage zuvor geschlüpft waren. Auch diese größeren Nauplien wurden von den jungen $\mathrm{He}-$ ringslarven gefressen.

b. Nahrungsaufnahme und Verdauungsgeschwindigkeit. Etwa 4 Tage nach dem Schlüpfen begannen die Heringslarven zu fressen, unabhängig davon, ob noch Reste des Dottersacks vorhanden waren. Die Nährtiere wurden in 1-2 Minuten bis an das anale Ende des Darmtrakts geschoben. Nach der Fütterung konnte man im Darm der lebenden Larve oftmals 5-10 Nährtiere erkennen. Bei Larven > $20 \mathrm{~mm}$ Körperlänge verliert der Darm seine Durchsichtigkeit, so daß keine Beobachtungen über den Darminhalt in vivo gemacht werden konnten. Die größeren Tiere, die kurz vor oder in der Metamorphose standen, fraßen besonders gierig. An einem ca. $35 \mathrm{~mm}$ langen Exemplar wurden etwa 100 Schnappbewegungen in den ersten 5 Minuten, nachdem frisches Wildplankton geboten worden war, gezählt. Schätzungsweise die Hälfte dieser Schnappbewegungen war erfolgreich.

Fast allen Autoren, die die Ernährung von Heringslarven in See studierten, ist der hohe Anteil leerer Därme in den Proben junger Larven aufgefallen (LEBour 1920, und später Mielck 1924, Marshall, Nicholls und Orr 1937, Bowers und Williamson 1951, HentsCHel 1950, Bhattacharyya 1957). Nur Hardy 1924 fand bei kleinen und großen Larven etwa die Hälfte der Därme gefüllt. Im allgemeinen ist der Füllungsgrad der Därme junger Larven sehr gering, erst bei größeren Larven wird eine starke Füllung des Darmes häufiger. 
Sehr große Mengen, wie sie z. B. OGulve 1927 beschrieb (211 Oithona similis in einer Larve von $45 \mathrm{~mm}$ Länge) sind aber zweifellos Ausnahmen. Die meisten der genannten Arbeiten geben Überblicke über die Anderungen in der Nahrungszusammensetzung, die im Laufe des Larvenlebens vom Dottersackstadium bis zur Metamorphose auftreten. Für kleine Larven scheint eine größere Vielfalt an Nährtieren typisch zu sein, was die Bedeutung des Wildplanktons als Futter bei Aufzuchtversuchen unterstreicht. In Anbetracht der starken Tagesperiodik in der Nahrungsaufnahme der Heringslarve (Hentsches 1950, Bhatrachanrya 1957) sind die Seeuntersuchungen nur schwer mit den Laborexperimenten zu vergleichen. Geht man von der Häufigkeit gefüllter Därme und ihrem Füllungsgrad aus, so läßt sich annehmen, daß in den Zeiten guten Planktonangebots die Larven im Versuch ebensogut oder besser fraßen als normalerweise in See.

Versuche über die Durchgangszeit der Nahrung sind bei Heringslarven leicht durchzuführen, da die Larven bei Dunkelheit nicht fressen und so die Nahrungsaufnahme kurz nach der Fütterung durch Ausschalten des Lichtes abgebrochen werden kann.

\section{Tabelle 4}

Verdauungsgeschwindigkeit von Heringslarven, die Anfang Mai geschlüpft sind Freßzeit = Zeitspanne von der Fütterung bis zum Ausschalten des Lichtes (d. h. Versuchsbeginn) $\mathrm{Wp}=$ Wildplankton, $\mathrm{A}=$ Artemia - Nauplien

\begin{tabular}{|c|c|c|c|c|c|c|}
\hline Datum & Temperatur & $\begin{array}{l}\text { Freßzeit } \\
\text { in Min. }\end{array}$ & $\begin{array}{r}<15 \mathrm{~mm} \\
\text { Lar } \\
\text { Verdauungs }\end{array}$ & $\begin{array}{l}>15 \mathrm{~mm} \\
\mathrm{t} \text { in Std. }\end{array}$ & Futter & $\begin{array}{l}\text { Füllungsgrad } \\
\text { b. Versuchs- } \\
\text { beginn }\end{array}$ \\
\hline 7.6 .60 & $9,0^{\circ} \mathrm{C}$ & 30 & $4,5-5,5$ & - & $W p+A$ & gut \\
\hline 8.6 .60 & $8,4^{\circ} \mathrm{C}$ & 80 & $5-7$ & - & $W_{p}+\mathrm{A}$ & gut \\
\hline 30.6 .60 & $10,1^{\circ} \mathrm{C}$ & 75 & 5,5 & 4,5 & $\mathrm{~W}_{\mathrm{p}}(+\mathrm{A})$ & mäßig \\
\hline 2.7 .60 & $8,4^{\circ} \mathrm{C}$ & ? & 5 & 4,5 & $W_{p}(+A)$ & gut \\
\hline 3.7 .60 & $10,6^{0} \mathrm{C}$ & ? & 4,5 & 4 & A & gut \\
\hline 6.7 .60 & $11,0^{\circ} \mathrm{C}$ & 180 & $>5$ & 4 & $W p+A$ & sehr gut \\
\hline 7.7.60 & $12,0^{\circ} \mathrm{C}$ & 50 & 6 & 5 & $\mathrm{~A}$ & sehr gut \\
\hline 8.7 .60 & $13,0^{\circ} \mathrm{C}$ & 40 & 4 & 3,5 & $W_{p}$ & gut \\
\hline 4.7 .60 & $13,5^{\circ} \mathrm{C}$ & 60 & 4 & 3,5 & $W_{p}+A$ & gut \\
\hline
\end{tabular}

Vor Versuchsbeginn wurden den Larven reiche Mengen von Wildplankton und/oder Artemia-Nauplien geboten. Nach 60-90 Minuten war der Darm in fast allen Larven etwa zu einem Drittel gefüllt.

Dann wurde das Licht ausgeschaltet und die Larven in den Becken in etwa stündlichen (später halbstündlichen) Abständen mit einer Taschenlampe inspiziert. Als Ende der Verdauungszeit wurde der Zeitpunkt benannt, zu dem nur noch wenige Larven kleine Nahrungsmengen in der Analregion des Darmes aufwiesen. In Tabelle 4 sind die Verdauungszeiten, die in den einzelnen Versuchen ermittelt wurden, zusammengestellt. Allgemein war auffallend, daß die Endabschnitte der Därme kleiner Larven längere Zeit gefüllt waren, als die der größeren Larven. Anscheinend ist der Darm der jungen Larve weniger leistungsfähig, obschon wahrscheinlich bei ihm die resorbierende und sezernierende Oberfläche relativ groß ist. Im Rahmen der geringen Temperaturunterschiede deutet sich eine Abhängigkeit der Verdauungsgeschwindigkeit von der Temperatur und dem Füllungsgrad des Darmes zu Versuchsbeginn an. Die Temperaturabhängigkeit der Verdauungszeit von Clupea pallasiiLarven untersuchte Kurata (1959), einige Beobachtungen an harengus-Larven machte Blaxter (1961). Seine Ergebnisse stimmen mit den auf Helgoland gewonnenen gut überein, während Kurata Verdauungszeiten von 12-20 Stunden feststellte. Nach Untersuchungen in See schätzte Hentsched (1950) die 
Durchgangszeit bei Heringslarven verschiedener Größe auf etwa 6 Stunden, die Larven waren in Wasser von $8-10^{\circ} \mathrm{C}$ gefangen worden.

\section{Wachstum und Entwicklung}

Die Versuche geben nur wenig Hinweise auf die Wachstumsgeschwindigkeit der Heringslarven. Hierfür gelten vor allem methodische Gründe: Es ist schwierig, die Länge der lebenden Larven im Becken zu bestimmen. Wir schätzten die Körperlänge der frei schwimmenden Larven durch den Vergleich mit einem ins Wasser gehaltenen Meßstab. Eine regelmäßige Entnahme repräsentativer Proben war bei der beschränkten Zahl der die ersten Wochen überlebenden Larven nicht möglich. Die im Becken gestorbenen Larven bieten kein zuverlässiges Bild von der Längenzusammensetzung der Larvenbevölkerung, auch waren sie oftmals postmortal geschrumpft. Diese Schrump-

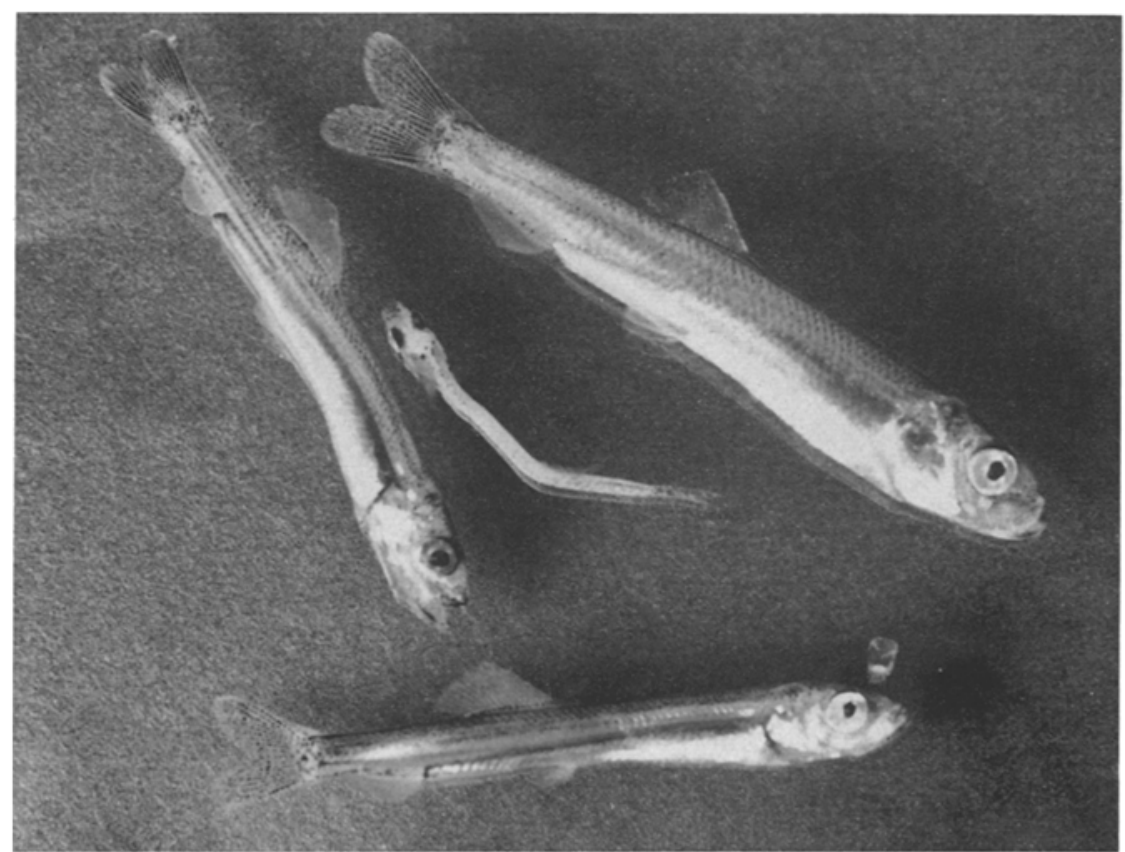

Abb. 6. Frischgetötete Heringe aus Becken 5 bei Abbruch der Aufzuchtversuche (phot. Dr. ZuegenmeIER)

fung, die während und unmittelbar nach dem Tode auftritt, kann eine Verkürzung der Larven bis zu 30\% der Gesämtlänge zur Folge haben. Ahnlich wie bei den früheren Versuchen in Aberdeen (BLAXTER 1961) waren die Längenunterschiede der Larven gleicher Herkunft und gleicher Lebensgeschichte sehr auffallend. Beispielsweise konnten in einem Becken nebeneinander Larven von $10-12$ und $20-25 \mathrm{~mm}$ Körperlänge beobachtet werden. Auch die kleinsten Larven, die wochenlang kaum größer wurden, fraßen gut, und so war eine Ursache für die Größenunterschiede nicht zu erkennen. Abb. 6 zeigt die letzten vier überlebenden Larven eines Kühlraum-Beckens, als am 26. 8. 60 die Versuche abgebrochen werden mußten. Die Totallänge der abge- 
bildeten Tiere betrug: $39 \mathrm{~mm}, 33 \mathrm{~mm}, 32 \mathrm{~mm}, 20 \mathrm{~mm}$, diese Larven waren etwa 110 Tage alt. Im Kühlraum erreichten die am schnellsten wachsenden Larven am Ende des 1. Larvenmonats 12-15 mm Körperlänge, einen Monat später waren sie etwa $20 \mathrm{~mm}$ lang, nach 3 Monaten hatten sie $30-35 \mathrm{~mm}$ erreicht und begannen zu metamorphisieren.

Meyer (1878b) erzielte bei seinen Aufzuchtversuchen, die bei höherer Temperatur stattfanden, fast die gleichen Zuwachswerte. Er wies darauf hin, daß Larven gleicher Herkunft, die in der Schlei aufwudsen, einen besseren Zuwachs zeigten. Er vermutete, daß das Nahrungsangebot im Zuchtbecken zeitweilig viel schlechter war als in See. In Scriacus (1939) Versuchen wuchsen die Larven etwa doppelt so schnell wie in unsern Experimenten. Das Wachstum der auf Helgoland gehaltenen Larven war aber immer noch erheblich besser als das der Larven der Herbst- und Frühwinterlaicher in der südlichen Nordsee (BückManN 1950), wo niedrige Wassertemperaturen und geringe Plankton-Konzentration hemmend wirken.

Die frisch gestorbenen und einige getötete Larven boten die Möglichkeit, die Entwicklung der Larven in Beziehung zur Körpergröße zu setzen (Tabelle 5). Ein Vergleich von Tabelle 5 mit den von Blaxter (1961, Tabelle IV)

Tabelle 5

Entwicklungsstadien der künstlich erbrüteten Larven deutscher Küstenheringe

Totallänge

Entwicklungsstadium

\author{
Schlüpfen \\ Ende des Dottersackstadiums \\ Dorsalflosse wird gebildet, Hypuralia werden angelegt \\ Schwanz wird aufwärts gebogen \\ Analflosse wird gebildet, Hypuralia färbbar mit Alizarin \\ caudale Hämalbögen färbbar \\ Wirbelzentren in der Körpermitte färbbar \\ 1. Zentrum des Urostyls färbbar \\ Beginn der Metamorphose \\ Metamorphose beendet
}

beschriebenen Verhältnissen zeigt, daß die Larven der Küstenheringe nicht deutlich weiterentwickelt sind als Clyde-Heringe gleicher Länge.

Schnakenbeck (1929) beschreibt auf Grund einiger fixierter Larvenproben die Entwicklungsstadien verschiedener Heringsgruppen. Danach tritt bei Larven der nordwestlichen Nordsee die Rückenflosse bei $9-10 \mathrm{~mm}$ Totallänge und die Afterflosse bei $15-16 \mathrm{~mm}$ auf, Larven der südwestlichen Nordsee sind anfangs weniger differenziert, später erfolgt bei ihnen dic Differenzierung relativ zur Körperlänge schneller. BRIDGER (1956) teilte die in der Flämischen Bucht und in der Straße von Dover gefangenen Heringslarven in 5 Entwicklungsstadien ein. Bei 8-9 mm Körperlänge ist der Dotter weitgehend resorbiert und die Flossenstrahlen der Caudalflosse werden erkennbar, bei 11-12 mm kommt die Dorsalflosse hinzu, bei $16-18 \mathrm{~mm}$ die Analflosse, bei 23-25 mm die Ventralflosse. Hempel (unveröffentlicht) fand bei Seeproben aus dem gleichen Gebiet eine größere Variation in den Beziehungen zwischen Körperlänge und Entwicklungsstadium. Die Unterschiede können z. T. durch unterschiedliche Schrumpfung der einzelnen Larven entstanden sein. Die Larven des vor Plymouth laichenden Herings zeigten eine abweichende Entwicklung (Lerour 1921). Der Dotter wurde spät resorbiert (Reste waren bei einigen Larven von $12 \mathrm{~mm}$ noch erkennbar). Nach seiner vollständigen Resorption wurde die Dorsale gebildet $(9-12 \mathrm{~mm})$. Die weitere Differenzierung erfolgte langsam, die Metamorphose trat erst bei mindestens $48-50 \mathrm{~mm}$ Körperlänge ein. Mryer (1878 a, b) und HEINCKE (1878) untersuchten künstlich erbrütete oder in See gefangene Ostseeheringe. Die Larven des Frühjahrslaichers der Schlei zeigten bei etwa $10 \mathrm{~mm}$ die Rückenflosse, bei 15 bis $19 \mathrm{~mm}$ langen Larven wurden die Schwanz- und Afterflossen angelegt. Mit 25-28 mm traten die ersten Larven in das Übergangsstadium zur Metamorphose ein, diese war bei 37-44 mm abgeschlossen. Nach Meyer (1878 a) liegt das Ubergangsstadium dieser Heringe normalerweise 
bei 32-45 mm. Bei den Herbstheringen der westlichen Ostsee erfolgt die Metamorphose erst erheblich später ( 44 bis $>60 \mathrm{~mm}$ Körperlänge). Dies entspricht der Regel, daß eine niedrige Temperatur die Differenzierung stärker hemmt als das Wachstum. Bei der Verwendung von Seeproben zur Ermittlung des mittleren Metamorphosetermins können gewisse Fehler auftreten. Da sich mit der Metamorphose der Heringe ihre Fangbarkeit ändert, ist in der Größengruppe $35 \mathrm{~mm}$ der Anteil unmetamorphisierter Tiere in den Fängen oft weit größer als im Gesamtbestand. Harder (1952) gibt für die Larven des Nordsee-Bankherings $40-50 \mathrm{~mm}$ Standardlänge (d. h. 43-53 mm Totallänge) als Metamorphoselänge an. Manches spricht dafür, daß viele Larven dieses Bestandes schon bei einer geringeren Körperlänge metamorphisieren.

In den Versuchen war es möglich, die Metamorphose der Larven am einzelnen Tier zu verfolgen. Die Umwandlung von der Larve zum Junghering verlief bei $15^{\circ} \mathrm{C}$ in etwa 10 Tagen überraschend schnell. Das erste Anzeichen war eine zunehmende Pigmentierung der dorsalen Körperhälfte, dann wurde der Kopf, der bei der Larve dorsoventral abgeplattet ist, seitlich komprimiert, und gleichzeitig gewann auch der Körper die typische Heringsform. Dieser Eindruck wurde dadurch unterstrichen, daß die Dorsal- und Caudalflossen Pigment anlegten und der Bauch silbrig wurde. Einschneidende Anderungen im Verhalten konnten wir - abgesehen von einer größeren Kontinuität der Schwimmbewegungen - im Gefolge der Metamorphose nicht beobachten. Allerdings überlebten nicht genügend Tier das Larvenstadium, um eine Schwarmbildung zu ermöglichen. Auch die Jungheringe zeigten noch die Sförmige Wartestellung, bevor sie auf ein Beutetier zustießen.

\section{Sterblichkeit}

In den Erbrütungsversuchen ergaben sich vier Sterblichkeitsphasen:

1. Während der Eientwicklung wurde die Sterblichkeit besonders durch die Temperatur bestimmt (s. o., vgl. Abb. 1). Vor allem bei den Kieler Eiern wirkte hoher Salzgehalt $(>30 \%$ oo) ebenfalls ungünstig auf die Schlüpfrate. Auch zeigten die Versuche wiederum, daß dichter Besatz von Eiern auf den Glasplatten die Überlebensrate herabsetzt. Zahlreiche entsprechende Hinweise auf die Gefährlichkeit zu dichter Eiansammlungen finden sich in der Literatur. $\mathrm{Ob}$ hierbei immer ein $\mathrm{O}_{2}$-Mangel die entscheidende Rolle spielt und welche Bedeutung Anhäufungen von Stoffwechselprodukten haben, bedürfte weiterer Untersuchungen.

2. Die nächste Mortalitätsphase trat nach Ende des Dottersackstadiums auf. Während der Resorption des Dotters starben fast keine Larven. Dann aber gingen in den einzelnen Becken $70-100 \%$ aller Larven binnen einiger Tage ein. Das erste Symptom wurde von uns als die Goldaugen-Krankheit (golden eye-disease) bezeichnet. Die moribunden Larven standen kopfunter im Wasser, und die Augen reflektierten golden infolge der abnormen Körperhaltung. Die Todesursache war höchstwahrscheinlich der Nahrungsmangel. Einerseits fehlte es in einigen Becken an geeignetem Zooplankton in ausreichender Menge, und andererseits waren die Becken z. T. anfangs übervölkert mit Heringslarven. Dipl. Biol. Flüchter (schriftliche Mitteilung) verglich im Rahmen seiner noch nicht abgeschlossenen Untersuchungen zur Funktion des Kieferapparates des Herings gesunde und moribunde Larven nach der Resorption des Dotters. Er fand bei den moribunden Larven keine Hinweise auf eine abnorme Entwicklung des Schädels oder Kiefers. Sie zeigten dieselben Kieferbewegungen wie solche, die gefressen hatten. Wir können also nicht an- 
nehmen, daß diese Larven aus morphologischen Gründen verhungern mußten.

Eine größere Anzahl von Larven mit Goldaugen-Krankheit wurde an Herrn J. J.ZujLstra, Ijmuiden, geschickt. Es zeigte sich, daß sie den Larven glichen, die im Dezember 1959 im Laichgebiet des Downsherings gefangen worden waren und durch geringe Körpergröße auffielen. Im Winter 1959/60 war das Zooplankton im Kanaleingang sehr arm, und gleichzeitig herrschten relativ hohe Wassertemperaturen. Die holländischen, englischen und deutschen Seeuntersuchungen trafen nur eine sehr geringe Anzahl von Heringslarven in der Umgebung der Laichgebiete und später in den Aufwuchsgebieten der Deutschen Bucht an. Vermutlich ist die Heringsbrut in See verhungert. Auch im Seegebiet der Doggerbank hatte ZujLstra im Herbst 1959 verhungernde Larven beobachtet (schriftliche Mitteilung). Solerm (1942) fand erhebliche Mengen kleiner, toter Heringslarven in Planktonfängen bei norwegischen Laichplätzen. Er nimmt an, daß diese Larven nach der Resorption des Dotters verhungert waren.

Wie bei verschiedenen Versuchen früherer Autoren trat als weitere, allerdings weniger schwerwiegende Todesursache in den meisten Becken die "Gaskrankheit" auf. Larven mit Gasblasen im Darm trieben an der Wasseroberfläche. Es handelte sich dabei vorwiegend um Larven, die bereits gut gefressen hatten, d. h. die erste kritische Phase bereits überstanden hatten.

80-100 Tagesgrade nach dem Schlüpfen waren Larven, die Luftblasen geschluckt hatten, $8,5 \mathrm{~mm}$ lang, während Hungerlarven, die dem gleichen Becken 5 Tage später entnommen wurden, im Mittel num $7,7 \mathrm{~mm}$ lang waren. Die Larven mit "Gaskrankheit" hatten zumindest die am Ende des Dottersackstadium erreichte Länge gehalten, die Hungerlarven waren dagegen wieder auf ihre Schlüpflänge zusammengeschrumpft. Am Ende des Dottersackstadiums düften diese Larven ebenfalls ca. $8,5 \mathrm{~mm}$ lang gewesen sein. In einigen Serien wurden Proben 60 und 70-80 Tagesgrade nach dem Schlüpfen gemessen. In drei von vier Fällen waren die älteren Larven etwas kleiner als die zugehörigen jüngeren. Allgemein scheinen Heringslarven stärker als ältere Fische bei Hunger zu schrumpfen.

Die,, Gaskrankheit" wurde nur in denjenigen Becken beobachtet, in denen Luftblasen auf der Oberfläche flottierten. Diese Luftblasen traten durch das Aufheizen des mit Luft gesättigten Seewassers auf, sie hielten sich in der Kahmhaut der Wasseroberfläche. Durch Kühlung des Wassers und Reinigung der Oberfläche ließ sich die "Gaskrankheit“ in den einzelnen Becken bekämpfen. Im kältesten Becken, in dem die Luftblasen fehlten, wurde keine Larve mit Gasblasen im Darm beobachtet. In den anderen Becken begann die "Gaskrankheit" gegen Ende des Dottersackstadiums, d. h. wenn die Larven normalerweise anfangen zu fressen. Sснасн (1939) nahm an, daß feine Luftbläschen durch die Schluckbewegungen des Oesophagus zusammen mit Seewasser in den Darm gelangen und sich dort vor dem After ansammeln. Wir vermuten dagegen, daß zumindest bei etwas älteren Larven das aktive Schnappen von Luftblasen, die an der Oberfläche treiben, eine erhebliche Rolle spielt. Larven, die größer als etwa $12 \mathrm{~mm}$ sind, scheinen unempfindlich gegen die "Gaskrankheit" zu sein. Nie traten größere Larven mit Gasblasen im Darm auf, was wahrscheinlich nicht ausschließlich auf die Reinigung der Beckenoberfläche zurückzuführen ist.

3. In den meisten Becken, die nun 20-40 Larven enthielten, starben während des ersten Monats nach Uberwindung der ersten kritischen Phase und der „Gaskrankheit“ fast keine Larven. Als die Tiere eine Länge von etwa 16-20 Millimeter erreicht hatten, setzte aber eine zweite Mortalitätsphase ein, der die meisten Larven zum Opfer fielen. Sauerstoffmangel und eine allgemeine Ver- 
giftung mit Abbauprodukten des Phytoplanktons können nicht die alleinigen Ursachen dieser Sterblichkeit gewesen sein, da in jedem der Becken einige Larven überlebten und die Verluste nicht plötzlich auftraten, sondern sich über mehrere Wochen hinzogen. Allerdings war die Sterblichkeit am stärksten zur Zeit der großen Phytoplankton-Wucherung mit ihrem Mangel an geeigneter Nahrung. Es liegt nahe, daß geschwächte Larven in dieser Zeit gehäuft starben und nur die kräftigsten überlebten. Eine Schwächung der Larven kann morphologisch bedingt sein. Die größeren Larven sind sehr aktiv und haben einen großen Sauerstoffbedarf. Ihre Kiemen sind aber noch nicht voll entwickelt (Harder 1954), und das Verhältnis zwischen Körpermasse und Körperoberfläche ist deutlich schlechter, abgesehen davon, daß sie die großen Oberflächen des Dottersackes und der Primordialflosse eingebüßt haben. Die größeren Larven sind aber sehr aktiv und daher auf eine reiche Sauerstoffversorgung angewiesen. Es ist auch denkbar, daß bei der allometrischen Entwicklung der Organe und Organsysteme Stönungen auftreten können. In einigen Larven war der hintere Abschnitt des Darmes enorm angeschwollen, was auf eine Mißfunktion des Verdauungssystems hindeutet. - In anderen Fällen scheint der Tod durch eine Überfütterung mit Artemia-Nauplien bedingt worden zu sein.

Kоттнаus (1939) hatte bei seinen Versuchen die Erfahrung gemacht, daß nur in Becken, die für einige Stunden vom direkten Sonnenlicht getroffen wurden, die Larven die Resorption des Dotters überlebten. Sснасн (1939) fand ähnliches. In unseren Versuchen ergab sich dagegen, daß Larven durchaus bei künstlichem Licht aufgezogen werden können. Die Larven überlebten sogar letztlich besser in den künstlich erleuchteten Becken als im Tageslichtversuch, wo allerdings höhere Temperaturen herrschten, die aber noch erheblich unter den Sommertemperaturen in den Küstengewässern der westlichen Ostsee und unter der von Marshall, Nicholls und Orr (1937) und Blaxter (1960) für die Larven des Clyde-Herings beschriebenen Letaltemperatur (26 bis $27^{\circ} \mathrm{C}$ bzw. $22-24^{\circ} \mathrm{C}$ ) lagen.

Später zeigten einzelne große Larven ein seltsames Verhalten, indem sie sehr schnell an der Wasseroberfläche schwammen, wobei die obere Kopfhälfte aus dem Wasser ragte. Mit kurzen Unterbrechungen wurde dies Verhalten tagelang beibehalten. Es war nicht unbedingt tödlich, aber zwei oder drei Larven starben - vermutlich vor Erschöpfung -, obwohl sie in den kurzen Pausen fraßen.

Die hier geschilderten Mortalitätsphasen führten dazu, daß nur etwa $5 \%$ der Larven, die das erste kritische Stadium überstanden hatten, die Metamorphose erreichten. Das entspricht $0,1-0,3 \%$ aller geschlüpften Larven.

\section{Diskussion}

Die Versuche auf Helgoland zeigen im Gegensatz zu älteren Auffassungen, daß Heringslarven bis zur Metamorphose in kleinen Becken und mit künstlichem Licht aufgezogen werden können. Damit wird die Durchführung von Aufzuchtexperimenten unter bestimmten, kontrollierten Umweltbedingungen erheblich erleichtert. Die sehr niedrige, in unseren Versuchen erzielte Uberlebensrate ist in See wahrscheinlich nichts Ungewöhnliches, dennoch müßte es möglich sein, durch Verbesserung der Aufzuchtbedingungen die 
Sterblichkeit erheblich zu senken: Wahrscheinlich empfiehlt es sich, den größeren Larven (>20 mm) größere Becken zu bieten, in denen sie bei schnellem Schwimmen nicht so häufig an die Wände stoßen. In größeren Becken bereitet aber die Aufrechterhaltung eines reichen Nahrungsangebots Schwierigkeiten. Einige Beobachtungen wiesen darauf hin, daß ein lebhafter Wasserstrom im Becken für die großen Larven günstig ist. Auf allen Stadien wäre ein besseres Angebot von geeignetem Plankton, vor allem von kleinen Organismen für die jungen Larven, die gerade den Dotter resorbiert haben, zu fordern. Die größeren Aufzuchterfolge auf Helgoland im Vergleich zu den Versuchen von BLAXTER (1961) sind wahrscheinlich auf die Versorgung mit Wildplankton zurückzuführen. Die Qualität des Wildplanktons wird aber naturgemäß immer schwanken, auch wird sich ein zeitweiliges Massenauftreten von unerwünschten Formen wie Phaeocystis und Noctiluca kaum ausschließen lassen.

In See ist die Konzentration geeigneter Nahrung für die Heringslarven meist erheblich geringer als in den Becken. Das mag einer der Hauptgründe für die hohe Mortalität der Fischbrut sein, auch wenn in früheren Untersuchungen nicht immer klare Beziehungen zwischen der Planktondichte und der Zahl der aufkommenden Fischlarven gefunden wurden. Neben der Dichte des Nährtierbestandes und der Anzahl der Feinde spielt wahrscheinlich die Kondition der Larven am Ende des Dottersackstadiums eine große Rolle für die spätere Nachwuchsziffer. Unter diesem Gesichtspunkt hat die Menge des Dotters, die Dauer des Dottersackstadiums und die Länge der Larven nach der Resorption des Dotters vermutlich erhebliche biologische Bedeutung. Unsere Versuche zeigen gemeinsam mit älteren Untersuchungen den Einfluß von Temperatur und Salzgehalt auf die Kondition der Larven. Erbrütung unter günstigen Temperatur- und Salzgehaltsbedingungen führt zu einer günstigen Relation zwischen Betriebs- und Wachstumsstoffwechsel, d.h. zu kräftigem Wachstum bis zum Ende des Dottersackstadiums. Solche Larven haben einen besseren Start für die Freßphase und bessere Überlebenschancen. Die Faktoren, die die Größe und Lebensfähigkeit der Heringslarven beim Schlüpfen und am Ende des Dottersackstadiums bestimmen, sollen in weiteren Versuchen analysiert werden. Dabei wird auch die Frage nach dem Einfluß der Größe und der Rassenzugehörigkeit der Eltern auf die Kondition der Larven behandelt werden.

\section{Zusammenfassung}

Im Frühjahr und Sommer 1960 wurden auf Helgoland die Aufzuchtversuche an Heringsbrut wieder aufgenommen. Als Elterntiere dienten Frühjahrslaicher aus der Elbmündung und der Kieler Bucht und eine Kreuzung Kieler Hering $\times$ Clyde-Hering.

Erbrütung und Aufzucht erfolgten vornehmlich in kleinen Steinzeug-Bekken $(120 \mathrm{l})$ mit Seewasserdurchfluß in einem temperaturkonstanten Raum mit diffuser, künstlicher Beleuchtung. Bei den Temperaturversuchen wurde die Brut bis zur Resorption des Dotters bei ca. $5^{0}, 8^{0}, 11^{0}$ und $14^{0} \mathrm{C}$ gehalten. Später wurde in allen Becken die gleiche Temperatur angestrebt, die in den folgenden Monaten von $8^{\circ}$ auf $14^{\circ} \mathrm{C}$ anstieg.

Bei Verlassen des Eies waren die kalt erbrüteten Larven länger und verfügten über weniger Dotter als die warm erbrüteten. Am Ende des Dottersack- 
stadiums hatten aber die bei $8^{\circ} \mathrm{C}$ gehaltenen Larven die größte Körperlänge erreicht.

In einer weiteren Versuchsserie wurde die Brut gleicher, konstanter Temperatur, aber verschiedenem Salzgehalt ausgesetzt. Die bei $15 \%$, d. h. unter annähernd isotonischen Bedingungen gehaltenen Larven waren sowohl beim Schlüpfen als auch am Ende des Dottersackstadiums am längsten. Bei hohen Temperaturen $\left(>11^{\circ} \mathrm{C}\right)$ war die Schlüpfrate der Eier stark herabgesetzt. Die optimale Befruchtungs- und Schlüpfrate der Brut des Küstenherings lag im Gegensatz zum Nordsee-Bankhering im Bereich niedriger Salzgehalte (15 bis $20 \%$.

Bezüglich der Größe und der Schlüpfrate konnten keine Unterschiede $z$ wischen den Kiel $\times$ Kiel- und Kiel $\times$ Clyde-Larven festgestellt werden.

Bei der Aufzucht traten zwei Phasen hoher Sterblichkeit auf. Die Mehrzahl der Larven starb bald nach der Resorption des Dotters, wahrscheinlich infolge unzureichender Ernährung. Die besten Oberlebensraten wurden bei einem großen Angebot von frischgefangenem Zooplankton vermischt mit Artemia-Nauplien erzielt. Als weitere Todesursache junger Larven wurde das Auftreten von Gas im Darm beobachtet. Durch sorgfältige Beseitigung der Luftblasen von der Wasseroberfläche ließ sich diese Krankheit vermeiden.

Nach einigen Wochen trat eine zweite Mortalitätsphase auf, der meist Larven von etwa $16-20 \mathrm{~mm}$ zum Opfer fielen. Die Anfälligkeit dieser Larven hängt vielleicht damit zusammen, daß bei ihnen die Kiemen noch nicht funktionstüchtig sind, das Verhältnis von Körpermasse zu -oberfläche aber immer ungünstiger wird.

Etwa 5\% der Larven überstanden auch diese Mortalitätsphase $(0,1$ bis $0,3 \%$ aller geschlüpften Larven), sie legten bei $25 \mathrm{~mm}$ Körperlänge Wirbel an und metamorphisierten, als sie $30-35 \mathrm{~mm}$ lang und 3-4 Monate alt waren. Trotz der hohen Verluste konnten die Versuche zeigen, daß es möglich ist, Heringslarven unter kontrollierten Bedingungen in kleinen Becken und bei künstlicher Beleuchtung bis zur Metamorphose aufzuziehen.

Abschließend werden einige mögliche Verbesserungen in der Aufzuchttechnik genannt und die biologische Bedeutung der Konditionsunterschiede der Larven aus den verschiedenen Erbrütungsexperimenten kurz diskutiert.

\section{Summary}

Rearing experiments on herring were done using eggs and sperm from spring spawing herring of the Elbe estuary and Kiel Bight. A successful cross fertilization was also made between the eggs of a Kiel female and the sperm of a Scottish (Clyde) male herring, the sperm having been frozen for about six weeks.

The eggs were incubated and the larvae reared at temperatures of about 5 , 8,11 and $14^{\circ} \mathrm{C}$ in 1201 earthenware tanks with a sea water circulation, the apparatus being contained in a constant temperature room with artificial lighting. The temperatures were equalised in the tanks after the larvae had resorbed their yolk sacs and the temperature was then allowed to rise slowly from about $8^{\circ} \mathrm{C}$ to $15^{\circ} \mathrm{C}$ during the rearing phase.

At high temperatures (greater than $11^{\circ} \mathrm{C}$ ) the percentage hatching was much reduced. At hatching the larvae incubated at the lower temperatures 
tended to be longer and have less yolk than those larvae hatched at higher temperatures. At the end of the yolk sac stage the larvae kept at $8^{\circ} \mathrm{G}$ were longest.

No differences in size or percentage hatching were observed between the Kiel $\times$ Clyde cross and its Kiel $\times$ Kiel control.

In further experiments eggs and larvae were kept at a constant temperature but at different salinities. The highest percentage fertilization and hatching was found in a salinity of $15-20 \%$ (in contrast to that of North Sea Banks herring). The larvae were longest both at hatching and at the end of the yolk sac stage when kept in a salinity of $15 \%$ (isotonic conditions).

There were two main phases of mortality during rearing. Most larvae died at the end of the yolk sac stage probably due to inadequate suitable food. The best survival was found in tanks where the larvae were fed on wild plankton and Artemia nauplii. Another cause of death was swallowing of air bubbles. This mortality was reduced by careful removal of air bubbles from the water surface and by keeping the surface very clean.

After this initial phase of mortality there was good survival for some weeks until the larvae reached a length of $16-20 \mathrm{~mm}$. The cause of death at this stage might have been due to respiratory difficulties caused by the gills not yet being functional and by the increasingly unfavourable relationship between body area and volume.

About $5 \%$ of larvae which survived beyond the yolk sac stage (or 0,1 to $0,3 \%$ of hatched larvae) developed vertebrae at a length of $25 \mathrm{~mm}$ and metamorphosed when $30-35 \mathrm{~mm}$ long and 3-4 months old.

These experiments show that it is possible to rear herring larvae to metamorphosis using controlled conditions in small tanks and with artificial light.

Means of improving our rearing techniques are given and the biological implications of the differences in size of herring larvae reared under different conditions are discussed.

\section{Literaturverzeichnis}

Bhattacharyya, R. N., 1957: The food and feeding habits of larval and postlarval herring in the northern North Sea. Mar. Res. Scot. 3, 1-14.

Blaxter, J. H. S., 1955: Herring Rearing-I. The Storage of Herring Gametes. Mar. Res. Scot., No. 3.12 p.

Blaxter, J. H. S., 1956: Herring Rearing-II. The Effect of Temperature and other Factors on Development. Mar. Res. Scot., No. 5. 18 p.

Blaxter, J. H. S., 1957: Herring Rearing-III. The Effect of Temperature and other Factors on Myotome Counts. Mar. Res. Scot., No. 1.16 p.

B laxter, J. H. S., 1960: The effects of extremes of temperature on herring larvae. J. Mar. Biol. Assoc. U. K. 39 (3), 605-608.

Blaxter, J. H. S., 1961: Herring Rearing-IV. Rearing beyond the yolk sac stage. (In Vorbereitung).

Bridger, J. P., 1956: On day and night variation in catches of fish larvae. J. Cons. Int. Explor. Mer, 22 (1), 42-57.

Bowers, A. B. \& Williamson, D. I., 1951: Food of larval and early post-larval stage of autumn-spawned herring in Manx waters. Rep. Mar. Biol. Stat. Pt. Erin 63, 17-26.

Bü $c k m a n n, A$, 1950: Die Untersuchungen der Biologischen Anstalt über die Okologie der Heringsbrut in der südlichen Nordsee. Helgol. Wiss. Meeresunters. 3, 1-57.

Dannevig, A. \& Hansen, S., 1952: Factors involved in Hatching and Rearing fish eggs and larvae. Fisk, dir. Skrifter, Havundersøk. 10, 1.

Gillbricht, M., 1959: Fluchtentafeln zur Bestimmung des Salzgehaltes mit Hilfe des Seewasseraräometers. Helgol. Wiss. Mecresunters. 6, 239-240. 
Harder, W., 1952: Zur postembryonalen Entwidklung des Darmes beim Hering (Clupea harengus L.). Z. Anat. u. Entwicklungsgesch. 116, 379-398.

Harder, W., 1954: Die Entwicklung der Respirationsorgane beim Hering. Z, Anat. u. Entwicklungsgesch. 118, 102-123.

Hardy, A. C., 1924: The herring in relation to its animate environment; pt. I. The food and feeding habits of the herring with special reference to the East Coast of England. Fishery Investig. Ser. II, 7 (3), 1-53.

Heincke, F., 1878: Die Varietäten des Herings. Jber. Comm. wiss. Unters. Meere Kiel, IV-VI, 4I-132.

Hempe1, G., 1953: Die Temperaturabhängigkeit der Myomerenzahl beim Hering (Clupea harengus L.). Naturwissensch. 40, 467/68.

Hempel, G. \& Blaxter, J. H. S., 1961: The experimental modification of meristic characters in herring (Clupea harengus L.). In Vorbereitung für J. Cons. int. Explor. Mer.

Hentsche1, E., 1950: Die Nahrung der Heringslarven. Helgol. Wiss. Meeresunters. 3, $59-81$.

Holliday, F. G. T. \& Blaxter, J. H. S., 1960: The effects of salinity on the developing eggs and larvae of the herring. J. Mar. Biol. Assoc. U. K. 39 (3), 591-604.

Kändler, R. \& Dutt, S., 1958: Fecundity of Baltic Herring. Rapp. Proc. Verb. Int. Explor. Mer 143, 99-108.

Kotth a u s, A., 1939: Zuchtversuche mit Heringslarven. Helgol. Wiss. Meeresunters. 1, 349 bis 358 .

Kupffer, C., 1878: Uber Laichen und Entwicklung des Herings in der westlichen Ostsee. Jber. Comm. wiss. Unters. Meere Kiel IV-VI, 23-35.

Kurata, H., 1959: Preliminary Report on the rearing of Herring Larvae. Bull. Hokk. Regional Fish. Res. Lab. 20.

L ebour, M. V., 1920: The food of young fish III. J. Mar. Biol. Ass. U. K. 12, 261-324.

Lebour, M. V., 1921: The larval and post-larval stages of the Pilchard, Sprat and Herring from Plymouth district. J. Mar. Biol. Assoc. U. K. 12 (3), $417-457$.

Marshall, S. M., Nicholls, A. G. \& Orr, A. P., 1937: On the growth and feeding of the larval and post-larval stages of the Clyde Herring. J. Mar. Biol. Assoc. U. K. 22, $245-267$.

McMynn, R. G. \& Hoar, W.S., 1953: Effects of salinity on the development of the Pacific herring. Canad. J. Zool. 31 (4), 417-432.

Meyer, H. A., 1878a: Beobachtungen über das Wachsthum des Herings im westlichen Theile der Ostsee. Jber. Commiss. Wiss. Unters. Meere Kiel, IV-VI, 227-250.

Meyer, H. A, 1878b: Biologische Beobachtungen bei künstlicher Aufzucht des Herings der westlichen Ostsee. Wiegandt, Hempel \& Parey, Berlin.

Mielck, W., 1924: Heringslarven, Eier und Larven anderer Fische und Nahrung der Larven in der westlichen Nordsee im Oktober 1922. Ber. Dtsch. Wiss. Komm. Meeresforsch. 1. $209-248$.

Ogilvie, H. S., 1927: Observations on the food of post-larval herring from the Scottish Coast. Fish. Bd. Scot. Sci. Invest. 1927, 1, 1-10.

Schach, H., 1939: Die künstliche Aufzucht von Clupea harengus. Helgol. Wiss. Meeresunters. 1, 359-372.

Schnakenbeck, W., 1929: Entwicklungsgeschichtliche und morphologische Untersuchungen am Hering. Ber. Wiss. Komm. Meeresforsch. 5, 23-78.

Soleim, P. A., 1942: Årsaker til rike og fattige Ärganger av sild. Fiskeridir. Skrifter 7, H. 2.

Vlasblom, A. G., 1959: Laboratory rearing of mussel and oyster larvae from egg to veliger. ICES CM. 1959, ShelIfish Comm. Paper No. 98 (Ms).

Volodin, V. M., 1956: (Embryonic development of the autumn Baltic Herring and their oxygen requirement during the course of development.) Voprosi lkhtiologii 7, 123 bis 133 (russisch). Englische Übersetzung in Fish. Res. Bd. Canada, Translation Series No. 252, St. Andrews, N. B. 1960. 